\title{
Benchmark CFD Validation Data for Surface Combatant 5415 in PMM Maneuvers - Part II: Phase-averaged Stereoscopic PIV Flow Field Measurements
}

\author{
Hyunse Yoon ${ }^{1}$, Joseph Longo $^{1}$, Yasuyuki Toda ${ }^{2}$, and Frederick Stern ${ }^{1 *}$ \\ ${ }^{1}$ IIHR - Hydroscience\& Engineering, the University of Iowa, Iowa City, IA 52242, U.S.A. \\ ${ }^{2}$ Department of Naval Architecture \& Ocean Engineering, Osaka University, Osaka, Japan
}

\begin{abstract}
Part II of this two-part paper presents CFD benchmark local flow field measurements for a surface combatant in dynamic planar-motion-mechanism (PMM) maneuvers. Phase-averaged stereoscopic particle-image-velocimetry (PIV) measurements are performed for pure sway and pure yaw maneuvers. The geometry is DTMB model 5512, a $3.048 \mathrm{~m}$ geosim of DTMB model 5415. The experiments aredone in a $100 \mathrm{~m} \times 3 \mathrm{~m} \times 3 \mathrm{~m}$ towing tank. The measurement system is a custom-designed towingtank maneuvering test flow-mapping system, which features a PMM for captive model testing with an integrated stereoscopic PIV. Statistical convergence error is defined and monitored by using the confidence intervalsof the mean and variance. The quality of data is also assessed by following standard uncertainty assessmentprocedures. The present experimental dataare cross-referenced with computational simulations for identification of the major vortices in the flow. The core locations and trajectories of the major vortices are traced and the flow variables at the core locationsare analyzed.The tests of both Parts I and II are sufficiently documented and detailed to be useful as experimental benchmark data for validation of computational fluid dynamicscodes.Part I presents the force/moment/motion measurements.
\end{abstract}

Keywords: Stereoscopic particle-image-velocimetry; Ship maneuvering; Planar motion mechanism; Statistical convergence; Uncertainty assessment; Vortex core analysis

\footnotetext{
${ }^{*}$ Corresponding author: Frederick-stern@uiowa.edu; (319) 335-5215.
} 


\section{Nomenclature:}

$A_{n}, \theta_{n} \quad$ the $n$-th order harmonic amplitude and phase

$\mathrm{B}_{\mathrm{WL}} \quad$ breadth of the hull

$f \quad$ PMM frequency $\left(\mathrm{s}^{-1}\right)$

Fr $\quad U_{\mathrm{C}} / \sqrt{\mathrm{g} L}$, Froude number

g gravitational acceleration $\left(=9.81 \mathrm{~m} / \mathrm{s}^{2}\right)$

$\mathrm{H}_{\mathrm{trj}} \quad$ height of vortex core trajectory

I $\quad u / U$, turbulence intensity based on mean velocity

$I_{\%} \quad \sqrt{2 k / 3} \times 100$, percent turbulence intensity based on $U_{\mathrm{C}}$

$k \quad(u u+v v+w w) / 2$, turbulent kinetic energy

$L \quad$ model length between perpendiculars (equivalent to $L_{P P}=3.048 \mathrm{~m}$ )

Re $\quad U_{\mathrm{C}} L / v$, Reynolds number

$t \quad$ time $(\mathrm{sec})$

$T \quad$ period of one PMM cycle $(\mathrm{sec})$

$\mathrm{T}_{\mathrm{m}} \quad$ mean draft of hull

$U_{i} \quad$ phase-averaged velocity component

$U_{\mathrm{C}} \quad$ towing carriage speed $(=1.531 \mathrm{~m} / \mathrm{s})$

$u_{i} u_{j} \quad$ Reynoldsstress components

$\mathrm{W}_{\mathrm{trj}} \quad$ width of vortex core trajectory

$x, y, z \quad$ ship-fixed, inertial coordinates

$x_{\mathrm{PMM}}, y_{\mathrm{PMM}} \quad$ PMM-fixed, non-inertial coordinates

$y_{\text {core }} \quad y$-coordinate of vortex core location

$z_{\text {core }} \quad z$-coordinate of vortex core location

$\gamma \quad$ PMM phase-angle in degree

$v \quad$ kinematic viscosity

$\psi \quad$ heading angle

$\omega_{x} \quad \partial W / \partial y-\partial V / \partial z$, axial vorticity

Abbreviations:

ABKV (AK) aft-body keel vortex

$\mathrm{BKV}(\mathrm{BK})$ bilge-keel vortex

FBKV (FK) fore-body keel vortex

FP forward perpendicular

FSV (FS) free surface vortex

SDV (SD) sonar-dome vortex

\section{Subscripts:}

C

$\mathrm{P}$

rms

$\mathrm{S}$ center-plane

portside

root-mean-square

starboard

Operators (for any variable $\boldsymbol{x}$ ):

$\bar{x}$

$\langle x\rangle$ spatial-average over a distance

time-average over one PMM cycle 


\section{INTRODUCTION}

Local flow ship-hydrodynamics experiments are useful for physics modeling and validation of computational fluid dynamics (CFD) codes, as used in CFD Workshops over the last 35 years (most recently, Stern et al., 2009 and Larsson et al., 2014). Double-body wind tunnel hotwire experiments provide the most detailed/accurate turbulence information since data acquisition rates are high. Towing tank Pitot tube and PIV experiments provide mean flow and for the latter turbulence information, including boundary layer and wake, propeller-hull interaction, forward speed diffraction, roll decay and planar motion mechanism (PMM) pure sway and yaw maneuvers. PIV systems have progressed from 2D (Irvine et al., 2013) to stereoscopic (herein) to tomographic (Yoon et al., 2014) using towed model-fixed systems. Noteworthy is the use of integrated experiments and computations whereby the simulations guide the experiments, experiments validate the simulations, and once validated the simulations fill in the sparse experimental data for physics diagnostics and explication.

Longo et al. (2004) first reviewed PIV for ship velocity fields. The $26^{\text {th }}$ and $27^{\text {th }}$ ITTC Specialist Committee on Detailed Flow Measurements (ITTC, 2011 and 2014) provided a comprehensive overview of measurements for ship propulsion and maneuvering and LDV, PIV and wave-elevation measurement systems and uncertainty analysis. Recent local flow measurements focused on onset and progression of vortices for large amplitude static drift for surface combatant 5415 using Tomographic PIV (Yoon et al., 2014), KVLCC2 using LDV and stereoscopic PIV (Abdel-Maksoud et al., 2015) and Delft catamaran using stereoscopic PIV (Broglia et al. 2015).

The present experimentsareconducted aspartof aninternationalcollaborationwithFORCETechnology, Denmark, and CNR-INSEAN, Italy, under theauspicesofthe24th-25th International Towing Tank Conference (ITTC) Maneuvering Committee (MC) fordevelopment/applicationofuncertaintyanalysisproceduresandassessmentofscaleeffectsandfacilitybiases. Part I (Yoon et al., 2015) of this two-part paper provides the benchmark CFD validation force / moment / motion data for surface combatant 5415 in both static and dynamic PMM maneuvers and the present Part II provides the phase-averaged stereoscopic PIV flow field measurements for two specific dynamic maneuvering cases from Part I. The data from both Parts I and IIwereused as a test case for CFD validation at the SIMMAN (Workshop on Verification and Validation of Ship Manoeuvring Simulation Methods) 2008 and 2014. 


\section{EXPERIMENTAL METHODS}

\subsection{Facility and model}

The tests are conducted at the IIHR towing tank (Fig. 1 in Part I provides a rendering of the towing tank facility and equipment setup). The tank is $100 \mathrm{~m}$ long, $3 \mathrm{~m}$ wide and $3 \mathrm{~m}$ deep. A four-wheel carriage supports the planar motion mechanism (PMM) that is towed behind a drive carriage. The PMM carriage is also used as a point of attachment for model 5512 and the stereoscopic PIV (hereafter SPIV) system. Linear and rotational potentiometers are installed on the PMM to monitor and report sway and yaw positions, respectively.

The model geometry is DTMB model 5512, a 1:46.6 scale, $L=3.048 \mathrm{~m}$ (equivalent to the length between perpendiculars, $L_{P P}$ ), fiber-reinforced Plexiglas hull with block coefficient, $C_{\mathrm{B}}=$ 0.506. Table 5 in Part I provides the principal dimensions of the model. DTMB model 5512 is a geosim of DTMB model 5415 that is a 1:24.8 scale, $5.72 \mathrm{~m}$ long model of the U.S. Navy's precontract design for a surface combatant (DDG-51) ca. 1980 with a sonar dome bow and transom stern. The model is unappended except for port and starboard bilge keels. To initiate transition to turbulent flow, a row of cylindrical studs is fixed on the model at $0.05 \mathrm{~L}$ downstream of forward perpendicular (FP), in accordance with recommendations by the $23^{\text {rd }}$ ITTC (ITTC, 2002).

\subsection{Coordinate systems and PMM motion}

Two right-handed Cartesian coordinate systems are used. The $x y z$-coordinate system is an inertial frame of reference fixed to the moving model and used for the PIV measurements. The origin of the $x y z$-coordinate system is at the intersection of FP, hull centerline and calmwater plane, with $x$ along the model centerline to downstream, $y$ to starboard, and $z$ upward. The $x_{\mathrm{PMM}} y_{\mathrm{PMM}}$-coordinate system is a non-inertial frame of reference fixed to the PMM and used to describe the PMM motions in the horizontal plane parallel to the calm-water surface. The origin of the $x_{\mathrm{PMM}} y_{\mathrm{PMM}}$-coordinate system is at the intersection of the tank centerline and the transverse axis of the PMM. The $x_{\mathrm{PMM}}$ axis is along the towing centerline to downstream and the $y_{\mathrm{PMM}}$ axis is to starboard. Part I provides the relationships between these two coordinate systems.

PMM motions are described by sway amplitude $y_{\text {max }}$, yaw amplitude $\psi_{\text {max }}$, PMM frequency $f$, and drift angle $\beta$ as 


$$
\begin{aligned}
& y_{\mathrm{PMM}}=-y_{\max } \sin (2 \pi f t) \\
& \psi=-\psi_{\max } \cos (2 \pi f t)+\beta
\end{aligned}
$$

in combination with the advance $x_{\mathrm{PMM}}=U_{\mathrm{C}} t$. The heading $\psi$ is the angle between the model centerline and the tank centerline.

\subsection{IIHR SPIV system}

The presentSPIV is a LaVision Inc. custom-designed and built underwater SPIV system. It consists of a $120 \mathrm{~mJ}$ dual-headNd:YAGlaser, enclosed mirror arm, submerged light-sheet generator, two stainless-steel modular camera enclosures and mirror modules, twoMegaPlusII ES2020 1600×1200 pixel cross-correlation cameras fitted withCanon EF $50 \mathrm{~mm}$ f/1.8 lenses and Birger Engineering lens controllers, and computer and software for data acquisition and reduction of PIV recordings. The lenses are controlled remotely from LaVision software with usersupplied inputs that pass through the Birger lens controllers to internal lens motors for focus and aperture adjustments. The camera bodies are equipped for remote Scheimpflug angle adjustments, again through LaVision software with user-supplied inputs. The laser, mirror arm, and light-sheet generator are fixed on a precision-ground aluminum plate to help steer the laser beam and maintain good alignment of the beams through the duration of the experiments.

The assembly of the SPIV with model 5512 is shown in Fig. 1. The SPIV system is assembled on aSanshin Seisakusho automated two-axis $(y, z)$ traverse that slides manually in $x$ on a 4-m multi-beam strongback. The laser and light sheet optics are arranged to deliver a vertical light sheet in the $(y, z)$ plane.Both camerasare arranged asymmetrically in submerged enclosures downstream of the light sheet to minimize wave and flow-field effects of the enclosures at the measurement area. The laser, light-sheet generator, and camera enclosures are assembled on a lightweight matrix of aluminum extrusions for adjustability and rigidity. Standoff distance between the light sheet generator and the measurement area center is $554 \mathrm{~mm}$. The cameras are arranged with equal standoff distances between the enclosures and the measurement area (644 $\mathrm{mm}), 33^{\circ}$ of separation between cameras, and a $22^{\circ}$ angle between camera $\# 1$ and the towing tank longitudinal axis to provide clearance with the model and avoid an extremely shallow angle between camera $\# 2$ and the cross plane. A minimum separation angle of $30^{\circ}$ between cameras is 
maintained per LaVision recommendations for a good stereoscopic configuration. The size of the field of view is approximately $100 \mathrm{~mm} \times 130 \mathrm{~mm}$ in width and height, respectively.

The SPIV system is calibrated in-situ by submerging and fixing a $30 \mathrm{~cm} \times 30 \mathrm{~cm}$ two-tier LaVision calibration plate in the plane of the light sheet where both camera field-of-views overlap. Single images from each camera are used to create a mapping function of the plate markers which is used later to reconstruct 3D velocity vectors from particle image pairs. The original calibration is refined three-to-four times iteratively with aLaVision ( $\mathrm{DaVis}$ ) self-calibration procedure to account for translational and rotational misalignment of the calibration plate with respect to the light sheet plane. Typically, a group of about 100 images is used for a self-calibration, where the images are captured while the cameras are towed in open water at $1.531 \mathrm{~m} / \mathrm{s}$ without the model attached.

Silver coated hollow glass sphere particles (SH400S33 by Potters Beads) are used as the seed material. The particles have a mean diameter of $14 \mu \mathrm{m}$ and an average density of 1.7 gram $/ \mathrm{cm}^{3}$. About $12 \sim 13$ grams ( 2 scoops) of particles are mixed well with water by using a motorized stirrer in a95-liter (25-gallon) size water tank. The mixture is discharged using a waterpump and through a perforated tube of $1-\mathrm{cm}$ diameter and $50-\mathrm{cm}$ length. Seeding runsare made at a speed of about $0.3 \mathrm{~m} / \mathrm{s}$ along the tank centerline after each carriage run. About 7.5 liters (2 gallons) of the mixture is discharged through each seeding run.

PIV analysisisdone withLaVisionDaVis v7.2 softwarein batch processes. The correlations are completed with one pass using $64 \times 64$ pixel interrogation windows followed by two passes using $32 \times 32$ pixel interrogation windows. Fifty percent overlap is used in the horizontal and vertical directions on all correlation passes. A high-accuracy Whitaker reconstruction of the vectors is used in the final pass. Vectors are range-filteredstation by station using median values and bands (upper and lower bounds) for $U, V$, and $W$. The median values are by assuming an average $U$ of about $0.75 U_{\mathrm{C}}$ or $1.15 \mathrm{~m} / \mathrm{s}$ inside the viscous region for all $x$-stations and an order-ofmagnitude smaller average $V$ and $W(0 \sim \pm 0.1 \mathrm{~m} / \mathrm{s}$ depending on the $x$-station $)$ typical for this kind of axially dominant flow. Since the flow is forced externally by the PMM, oscillations are expected for all velocity components. Thus, the upper and lower bounds (or the bands of \pm 0.5 $\sim \pm 1 \mathrm{~m} / \mathrm{s}$ depending on the $x$-station) for all velocity components are chosen iteratively and empirically to cover the velocity oscillations. A median filter followed, removing vectors if their magnitude is greater than two times the standard deviation value of their neighboring vectors. 
Spurious vectors are not replaced with interpolated values, and blank spots in the measurement area are not filled.

\subsection{Test conditions}

All tests are conducted at one towing speed $U_{\mathrm{C}}=1.531 \mathrm{~m} / \mathrm{s}$ corresponding to $\mathrm{Fr}=$ $U_{\mathrm{C}} / \sqrt{\mathrm{g} L}=0.280$. The model is first ballasted to asinkage $\left(\sigma=1.92 \times 10^{-3} L\right)$ and trim $(\tau=-$ $0.136^{\circ}$, positive bow up) corresponding to the dynamic sinkage and trim in straight-ahead towing $\mathrm{atFr}=0.280$, then fixed rigidly to a fixed-mount restraining the model from all free motions.

Table 6 in Part I presentsthe $U_{\mathrm{C}}, y_{\max }, \psi_{\max }, f$, and $\beta$ values used inEqs.(1)and(2) for pure sway and pure yaw motions. The drift angle $\beta$ is zero for both motions.For pure sway, the PMM is adjusted for a non-dimensionalsway velocity $v_{\max }=y_{\max } \omega / U_{\mathrm{C}}=0.174$, where $\omega=2 \pi f$, corresponding to an effectivedrift angle $\beta_{\max }=\tan ^{-1}\left(v_{\max }\right)=10^{\circ}$. The model heading is kept at straight-ahead by setting $\psi_{\max }=\beta=0$ in Eq.(2).For pure yaw, the PMM is adjusted for a nondimensionalyaw rate $r_{\max }=\psi_{\max } \omega L / U_{C}=0.3$. The lateral motion is set to satisfy the constraint $\tan ^{-1}\left(y_{\max } \omega / U_{\mathrm{C}}\right)=\psi_{\max }$ such that the model is always tangent to its path-line.

The cyclic frequency $\omega=2 \pi f$ value $\left(0.842 \mathrm{~s}^{-1}\right)$ is examined for unfavorable memory effect or tank resonance following the ITTC recommended procedures and guidelines 7.5-02-06-02 'Captive Model Test Procedures.'The non-dimensional frequency $\omega_{1}^{\prime}=\omega L / U_{\mathrm{C}}<4$ is associated with avoidance of non-stationary lift and memory effects, $\omega_{2}^{\prime}=\omega \sqrt{L / g}=0.15 \sim 0.2$ the tank resonance, and $\omega_{3}^{\prime}=\omega U_{\mathrm{C}} / \mathrm{g}<<0.25$ the unrealistic combinations of pulsation and translation. For the present study, $\omega_{1}^{\prime}=1.67, \omega_{2}^{\prime}=0.47, \omega_{3}^{\prime}=0.13$, where $\omega_{2}^{\prime}$ violates the criteria due to the limitation in the present PMM capacity.

Geometric restrictions are also examined with regard to avoiding restricted-water effects following the ITTC (2002) suggestions as described in Metcalf et al. (2006). The constraints includethe Froude number based on water depth $\operatorname{Fr}_{h}=U_{\mathrm{C}} / \sqrt{\mathrm{gh}} \leq 0.7$, the ratio of water depth to model draft $h / T \geq 4$, the ratio of tank width to model length $W / L \geq 0.35$, the ratio of tank width to the model width $W / B \geq 4$, and the ratio of waterway-section to the mid-body section $A_{t} / A_{M}=$ $W h / B T \geq 15$. For the present study, all the criteria are satisfied with $\operatorname{Fr}_{h}=0.28, h / T=23.1, W / L$ $=1, W / B^{*} \approx 5$, and $A_{t} / A_{M}^{*} \approx 110$, where the blockage constraints $\left(W / B^{*}\right.$ and $\left.A_{t} / A_{M}^{*}\right)$ are by identi- 
fying the maximum cross-sectional area $A_{M}^{*}=B^{*} T$ that the model passes through during one PMM cycle and by using $B^{*}=2 y_{\max }$.

The flow Reynolds number $\operatorname{Re}=U_{\mathrm{C}} L / v=3.88 \times 10^{6}$ for pure sway based on $13^{\circ} \mathrm{C}$ water temperature in the IIHR towing tank during the test, where $v$ is the kinematic viscosity of fresh water. For pure yaw, $\mathrm{Re}=4.31 \times 10^{6}$ based on an average water temperature of $17^{\circ} \mathrm{C}$, where the water temperature gradually varies between $11^{\circ} \mathrm{C}$ to $23^{\circ} \mathrm{C}$ during the test, beginning from late March through early December.

\subsection{Data acquisition and phase-averaging}

An IIHR-designed and -built PIV synchronizer is used to provide equally-spaced trigger pulses to the PIV computer in order to acquire PIV recordings at pre-settable, repeatable phase angles. This is achieved as the synchronizer monitored the analog output from the PMM sway potentiometer. When a predetermined voltage corresponding to $45^{\circ}$ on the rising side of the sway curve is reached, the synchronizer emits a burst of 8 and 32 Transistor-Transistor Logic (TTL) pulsesfor pure sway andpure yaw, respectively. Each TTL has a $200 \mu \mathrm{sec}$ pulse width and a time between triggers of $933 \mathrm{~ms}$ for pure sway and $233 \mathrm{~ms}$ for pure yaw. Since the PMM frequency is $f=0.134 \mathrm{~Hz}$, this enables PIV recordings at equally spaced phase locations, $\gamma=(n-1) \Delta \gamma$, where $n=1,2, \ldots, 8$ and $\Delta \gamma=45^{\circ}$ for the pure sway test and $n=1,2, \ldots, 32$ and $\Delta \gamma=11.25^{\circ}$ for the pure yaw test. For both tests, laser-sheet thickness is set roughly to $4 \mathrm{~mm}$ to achieve a good balance between image brightness while pushing particle transit times as high as practical to increase signal to noise ratio, i.e., lengthen pixel displacement. For this sheet thickness, a time interval $\Delta t=980 \mu$ s between two pulses of double-pulse laser is used such that the particle residence time (or the travel length) in the laser-sheet is about $25-30 \%$ of the sheet thickness and good correlations are observed on both cameras 1 and 2 . A series of parametric tests are done to refine and determine $\Delta t$.

In consideration of the symmetry in the hull motions, the present measurements are done only for the flow at the starboard side and the portside flow is mirrored from the starboard measurements at a matching phase. For example, the portside flow at $\gamma=0^{\circ}$ is the mirrored image of the starboard flow measured at $\gamma=180^{\circ}$ and so on.

PIV measurements are done for four non-dimensional ship-length stations $(x=0.135$, $0.235,0.735$, and 0.935 of $L)$ for pure sway and six stations $(x=0.135,0.335,0.535,0.735$, 
0.935 , and 1.035 of $L$ ) for pure yaw. The data acquisition occurs at $1.072 \mathrm{~Hz}$ for $20 \mathrm{sec}$ enabling about 20 PIV recordings in one carriage run for pure sway. Whereas, the data acquisition occurs at $4.288 \mathrm{~Hz}$ for $20 \mathrm{sec}$ enabling about $85 \mathrm{SPIV}$ recordings in one carriage run for pure yaw. Data acquisition is completed at several overlapping zones at each $x$-station in order to piece together a complete picture of the region of interest. For pure yaw, at least 100 carriage runsare performed for each zone to obtain enough recordingsat each phase to achieve convergence of the data.

The phase-averaged velocity component $U_{i}$ is computed using the following equation

$$
U_{i}=\sum_{n=1}^{N} U_{i, n} / N
$$

The index $i=1,2$, or 3 in Eq.(3)indicates the axial $U$, transverse $V$, and vertical $W$ velocity component, respectively. The Reynold stress tensor component $u_{i} u_{j}$ is computed using the following equation

$$
u_{i} u_{j}=\sum_{n=1}^{N}\left(U_{i, n}-U_{i}\right)\left(U_{j, n}-U_{j}\right) / N
$$

The indices $i, j=1,2$, or 3 of $u_{i} u_{j}$ indicate the axial $u$, transverse $v$, and vertical $w$ velocity fluctuation, respectively. InEqs.(3)and(4), $U_{i, n}$ or $U_{j, n}$ represents the instantaneous velocity data acquired from the $n$-th PIV recording. Turbulent kinetic energy $k$ is computed using the Reynolds normal stresses in Eq.(4) for $i=j$ as

$$
k=\frac{1}{2}(u u+v v+w w)
$$

Through the remainder of the present paper,all length variables $(x, y, z)$ are presented as non-dimensional with the ship length $L$, all velocity variables $(U, V, W)$ with the towing speed $U_{\mathrm{C}}$, and all Reynolds stress components $u_{i} u_{j}$ and the turbulent kinetic energy $k$ with the squared towing speed $U_{\mathrm{C}}^{2}$, unless stated otherwise.

\section{STATISTICAL CONVERGENCE}

Statistical convergence of thephase-averaged variablesis monitored. Any velocity variable $x=U_{i, n}$ or $U_{j, n}$ inEqs.(3) and (4)is assumeda stationary random variable of which mean $\mu_{x}$ and 
variance $\sigma_{x}^{2}$ do not change with time. Additionally, a normal distributionis assumed for the variable. The stationarity of data is tested by using a non-parametric statistical procedure known as the 'Reverse Arrangement Test.'The normality of data is examined by using a Chi-square goodness-of-fit test. Yoon et al. (2015) provide more details of the methodology for each test.

A statistical convergence error is defined by using the confidence interval for $\mu_{x}$ based on sample mean $\bar{x}$ and sample standard deviation $s_{x}$ as

$$
E_{\mathrm{SC}}(\%)=\frac{t_{n ; \alpha / 2}}{\sqrt{N}} \cdot \frac{s_{x}}{x_{\text {ref }}} \times 100
$$

In Eq.(6), $t_{n ; \alpha / 2}$ is the Student- $t$ variable of $n=N-1$ degrees of freedomand for a $100(1-\alpha)$ percentlevel of confidence. The $x_{\text {ref }}$ can be any meaningful reference velocity value to scale the error. Similar statistical convergence error canbe defined fora Reynolds normal stress $u_{i} u_{j}$ inEq. (4),by using the confidence interval for $\sigma_{x}^{2}$ based on the sample variances ${ }_{x}^{2}$ as

$$
\begin{gathered}
E_{\mathrm{SC}}^{\mathrm{U}}(\%)=\left(\frac{n}{\chi_{n ; 1-\alpha / 2}^{2}}-1\right) \cdot \frac{s_{x}^{2}}{s_{\mathrm{ref}}^{2}} \times 100 \\
E_{\mathrm{SC}}^{\mathrm{L}}(\%)=\left(1-\frac{n}{\chi_{n ; \alpha / 2}^{2}}\right) \cdot \frac{s_{x}^{2}}{s_{\mathrm{ref}}^{2}} \times 100
\end{gathered}
$$

where, $E_{\mathrm{SC}}^{\mathrm{U}}$ is the upper limit and $E_{\mathrm{SC}}^{\mathrm{L}}$ is the lower limit of the error at a $100(1-\alpha)$ percent level of confidence. The $\chi_{n ; 1-\alpha / 2}^{2}$ and $\chi_{n ; \alpha / 2}^{2}$ are the Chi-square variables. The $s_{\text {ref }}^{2}$ can be any meaningful reference velocity-squared value to scale the errors. The present study estimates the errors at a $95 \%$ level of confidence and uses $\alpha=0.05$ for all variables.

Fig. 2 presents $E_{\mathrm{SC}}$ and $\left(E_{\mathrm{SC}}^{\mathrm{U}}, E_{\mathrm{SC}}^{\mathrm{L}}\right)$ as a chartfor a few typical $s_{x} / x_{\text {ref }}$ and $s_{x}^{2} / s_{\text {ref }}^{2}$ values in parts (a) and (b), respectively. These charts providequick estimations of $E_{\mathrm{SC}}$ for a given sample size or the minimum size of a sample for a desired error level. Since $s_{x}$ is equivalentto the rootmean-squarevelocity fluctuation $u$, the parameter $s_{x} / x_{\text {ref }}$ corresponds to theflowturbulence intensity $I=u / U$ if the mean velocity $U$ is used as $x_{\text {ref }}$. For example, if a sample size $N=100$ is used to measure a flow of $I=0.1$, then the expected $E_{S C}$ is about $2 \%$ from the $s_{x} / x_{\text {ref }}=0.1$ line of the chart in Fig. 2(a). If an error less than $1 \%$ is desired from the same flow, then the minimum sam- 
ple size $N_{\min }=400$ is required. The $N_{\min }$ can also be obtained by rewriting Eq.(6)using $t_{n ; \alpha / 2}=2$ and assuming $N>>10$,

$$
N_{\text {min }}=\left(\frac{2 \cdot I}{E_{\mathrm{SC}} / 100}\right)^{2}
$$

where, $I$ is the turbulence intensity and $E_{S C}$ is the desired error in percent value.Similarly, $\left(E_{\mathrm{SC}}^{\mathrm{L}}\right.$, $\left.E_{\mathrm{SC}}^{\mathrm{U}}\right)$ can be estimated by using the chart in Fig. 2(b). Since $s_{x}^{2}$ is equivalent to the mean-square of velocity fluctuation $u u$, the parameter $s_{x}^{2} / s_{\text {ref }}^{2}=1$ if an isotropic turbulence is assumed, i.e., $u u=$ $2 k / 3$ and $s_{\text {ref }}^{2}=2 k / 3$ is used. For a sample of size $N=100$, the $\left(E_{\mathrm{SC}}^{\mathrm{L}}, E_{\mathrm{SC}}^{\mathrm{U}}\right) \approx(23 \%, 34 \%)$ from the chart. If an error less than $1 \%$ in $u u$ is desired, then $N_{\min } \approx 76,000$ is required.

A single point Pisselected near the local-peak $k$ location of a typical sample flow. A total number $N_{\text {total }}=270$ PIV recordings is collected from 103 carriage runs. All velocity components $(U, V, W)$ at $\mathrm{P}$ pass the 'Reverse Arrangement Test' with the total number of reverse arrangement $A=113 \sim 118$ for the mean and $A=70 \sim 97$ for the variance values from a sequence of $N$ $=20$ intervals, respectively. The acceptance region is $65<A \leq 125$ for $\alpha=0.05$ and the hypothesis that the variables display no trend is accepted at a 5\% level of significance. The variables also pass the normality test with $\mathrm{X}^{2}=15 \sim 16$ by using a class interval $K=15$. The acceptance regions is $\mathrm{X}^{2} \leq 21$ for $n=K-3=12$ and $\alpha=0.05$, thus the normality hypothesis is also accepted at a $5 \%$ level of significance.

The whole of the data points from the sample flow measurement aresplit into three groups based on the flow turbulence level, Group A $(k /[k] \leq 0.1)$, Group B $(0.1<k /[k] \leq$ $0.5)$, and Group $\mathrm{C}(0.5<k /[k] \leq 1.0)$, representing the regions where a fast, moderate, or slow convergence is expected, respectively. The $[k]=0.013$ is the range of $k$ values within the entire field of view combining all three groups. The area coverage by each group is $47 \%, 44 \%$, and $9 \%$, respectively. The actual vector number $N$ used foraveraging is $0.81,0.74$, and $0.63 N_{\text {total }}$ in averages for each group, respectively. The $N$ values are less than $N_{\text {total }}$ due to missing or rejected vectors. Herein, the $E_{\mathrm{SC}}$ values are presented in percent of $U_{\mathrm{C}}$ and the $\left(E_{\mathrm{SC}}^{\mathrm{L}}, E_{\mathrm{SC}}^{\mathrm{U}}\right)$ values are in percent of $2[k] / 3$, by using $x_{\text {ref }}=1$ and $s_{\text {ref }}^{2}=2[k] / 3$, respectively. Turbulence is low for group A with $I \leq 0.03$ and the error values are small with $E_{\mathrm{SC}}=0.3 \%$ and $\left(E_{\mathrm{SC}}^{\mathrm{L}}, E_{\mathrm{SC}}^{\mathrm{U}}\right)=(1.3 \%, 1.7 \%)$ in averages. Group B exhibits a mild turbulence intensity of $I=0.03 \sim 0.07$ and the error values are mod- 
erate with $E_{\mathrm{SC}}=0.7 \%$ and $\left(E_{\mathrm{SC}}^{\mathrm{L}}, E_{\mathrm{SC}}^{\mathrm{U}}\right)=(5.8 \%, 7.8 \%)$ in averages. Turbulence is the largest for group $\mathrm{C}$ with $I=0.07 \sim 0.09$ and the errors are also the largest with $E_{\mathrm{SC}}=1.3 \%$ and $\left(E_{\mathrm{SC}}^{\mathrm{L}}, E_{\mathrm{SC}}^{\mathrm{U}}\right)=$ $(15.2 \%, 21.1 \%)$ in averages. The local maximum errors are as high as $E_{\mathrm{SC}}=2.3 \%$ and $\left(E_{\mathrm{SC}}^{\mathrm{L}}, E_{\mathrm{SC}}^{\mathrm{U}}\right)$ $=(34.9 \%, 52.2 \%)$, which occurs near the point $\mathrm{P}$. Although not shown, the $V$ and $W$ velocities exhibit smaller $E_{\mathrm{SC}}$ than $U$ and the $v v$ and $w w$ stresses exhibit smaller $\left(E_{\mathrm{SC}}^{\mathrm{L}}, E_{\mathrm{SC}}^{\mathrm{U}}\right)$ than $u u$.

\section{UNCERTAINTY ASSESSMENT}

The UA follows the ASME PTC 19.1-2005 Standard (ASME, 2005). The ASME (2005) is a revision of the ASME PTC 19.1-1998 Standard (ASME, 1998). The procedure is based on estimates of systematic $\left(b_{R}\right)$ and random $\left(s_{R}\right)$ standard uncertainties for any result variable $R$, and their root-sum-square (RSS) combination to ascertain the combined standard uncertainty, $u_{R}$ $=\sqrt{b_{R}^{2}+s_{R}^{2}}$. Systematic uncertainties are estimated with considerations of elemental error sources for individual variables, whereas random uncertainties are estimated end-to-end. Finally, the combined standard uncertainty is expanded to a $95 \%$ level of confidence by multiplying an expansion factor $t_{95}$ such that $U_{R, 95}=t_{95} \cdot u_{R}$,termed as the 'expanded' uncertainty.

The estimation of $b_{R}$ usesa reference measurement $R_{\text {Ref }}$ as a calibration standard and the difference $\delta=R-R_{\mathrm{Ref}}$ is considered as the systematic error in $R$ and thestandard uncertainty is defined as

$$
b_{R}=\left(b_{\delta}^{2}+b_{R_{\mathrm{Ref}}}^{2}\right)^{\frac{1}{2}}
$$

The term $b_{R_{\text {Ref }}}$ in Eq.(10)is the systematic uncertainty in $R_{\text {Ref }}$ and

$$
b_{\delta}=\left[\bar{\delta}^{2}+\left(\frac{t \cdot s_{\delta}}{\sqrt{M}}\right)^{2}\right]^{\frac{1}{2}} / 2
$$

where, $t$ is the Student- $t$ variable for degrees of freedom $v=M-1$ and $95 \%$ confidence.The $\bar{\delta}$ and $s_{\delta}$ in Eq.(11)are the mean and standard deviation, respectively, of $\delta$ from a number of $M$ repeat measurements.

An open-water flow is measured by towing the PIV in pure yaw motion but with no model attached. A total of $M=3$ repeat tests is made with about 100 carriage runs for each test allowing $270 \sim 280$ samples. For $R=U$ and $V$ velocity components, theexpected values from the 
PIVmeasurements are essentially the coordinate transforms of the PMM velocities $v_{\mathrm{PMM}}=$ $\dot{y}_{\mathrm{PMM}}$ and $r_{\mathrm{PMM}}=\dot{\psi}$ to the ship-fixed coordinate system, where $y_{\mathrm{PMM}}$ and $\psi$ are as inEqs.(1)and(2), respectively, thus $R_{\mathrm{Ref}}=R_{\mathrm{Ref}}\left(y_{\max }, \psi_{\max }, f, t\right)$. The elemental uncertainties are $b_{y_{\max }}=B_{y_{\max }} / 2 L=1.6 \times 10^{-4}, b_{\psi_{\max }}=B_{\psi_{\max }} / 2=0.11^{\circ}, b_{f}=B_{\omega} / 4 \pi=5 \times 10^{-6} \mathrm{~Hz}$, and $b_{t}=$ $B_{t} / 2=5 \times 10^{-4} \mathrm{~s}$, where $B_{y_{\max }}=1 \mathrm{~mm}, B_{\psi_{\max }}=0.22^{\circ}, B_{\omega}=6.3 \times 10^{-5} \mathrm{sec}^{-1}$, and $B_{t}=0.001 \mathrm{sec}$ from a previous UA in Yoon et al. (2015). Through the propagations of these elemental uncertainties, $b_{R_{\mathrm{Ref}}}=0.005$ and 0.002 are obtained for $R=U$ and $V$, respectively.From the present measurements, $\bar{\delta}=-0.011$ and $s_{\delta}=0.003$ are observed for $U$ and $\bar{\delta}=0.009$ and $s_{\delta}=0.006$ for $V$, and from Eq.(11), $b_{\delta}=0.006$ for both variables by using $t=4$.3for $v=2$.Subsequently, $b_{R}=$ 0.008 for $U$ and $b_{R}=0.007$ for $V$ are calculated by using Eq.(10). Since a perfectly calm water is assumed, $R_{\text {Ref }}=b_{R_{\mathrm{Ref}}}=0$ are used for all other variables, from which $b_{R}=0.006$ for $W, b_{R}=$ $(0.00020,0.00011,0.00004)$ for $(u u, v v, w w)$ and $b_{R}=(0.00011,0.00001,0.00001)$ for $(u v, u w$, $v w)$.

The $s_{R}$ is computed with the standard multiple-test equation

$$
s_{R}=\sqrt{\left(\frac{\sum_{i=1}^{M}\left(R_{i}-\bar{R}\right)}{M-1}\right)} / \sqrt{M}
$$

where, $\bar{R}$ is the mean of a sample of $M$ realizations of variable $R$ and $R_{i}$ is the sample from the $i$ th realization. Three sets $(M=3)$ of repeated pure yaw tests are performed with the model attached, each test with more than 100 carriage runs. The measurement location is $x=0.135$. The PIV assembly is repositioned in the $(x, y, z)$ directions for each set to introduce a perturbation in the test setup. Each carriage run is made with a time-interval of about 12 minutes or longer and each test-set is done with a time-span of $4 \sim 5$ days including the PIV repositioning process.

Essentially, a large sample size is assumed in ASME (2005) and $t_{95}=2$ is used. However, the sample size is only 3 for the present study. Thus, an appropriate $t_{95}$ values is found by using an effective degrees-of-freedom using the Welch-Satterthwaite formula provided in the 'Non-mandatory Appendix B' of ASME (2005),

$$
v_{R}=\frac{\left(b_{R}^{2}+s_{R}^{2}\right)^{2}}{b_{R}^{4} / v_{b_{R}}+s_{R}^{4} / v_{s_{R}}}
$$


where, $v_{S_{R}}=M-1$ and $v_{b_{R}}$ are the degrees of freedom in $s_{R}$ and $b_{R}$, respectively. The ISO Guide (1995) recommends the approximation $v_{b_{R}}=\left(\Delta b_{R} / b_{R}\right)^{-2} / 2$, where the quantity in parentheses is an estimate of the relative variability of the estimate of $b_{R}$. For the present study, $v_{b_{R}}=$ 50 by assuming $\Delta b_{R} / b_{R}=0.1$ and $v_{S_{R}}=2$ for $M=3$ are used.

The measurement area is split into sub-regions by using a criterion based on the kinetic energy $K=\left(U^{2}+V^{2}+W^{2}\right) / 2$ value. The inner region is defined as the region where $K \leq 0.45$ representing the near field of the flow. The outer region is defined as the region where $K>0.45$ representing the far field of the flow. The UA values are averaged within each region. Table 1 summarizes the inner region results. For $(U, V, W)$, usually $b_{R}$ is dominant with the relative contribution about $30 \sim 80 \%$. An effective $v_{R}=15$ is used for these variables and $U_{R, 95}=2 \sim 3 \%$ of $U_{\mathrm{C}}$ is estimated by using $t_{95}=2.13$. The $U$ velocity exhibits the largest uncertainty value among these variables. For theturbulence variables, $s_{R}$ is dominant with the relative contribution usually larger than $90 \%$. The effective $v_{R}=2$ for these variables and $t_{95}=4.3$ is used. $U_{R, 95}=6 \sim 23 \%, 4 \sim$ $11 \%$, and $17 \%$ of $[k]$ are estimated for $(u u, v v, w w),(u v, u w, v w)$, and $k$, respectively, where $[k]=0.015$ is the range value of $k$ within the entire measurement area combining the inner and outer regions together. The largest uncertainty is for $u u$ among the normal stress components and for $u v$ among the shear stress components.For the outer region, $b_{R}$ is dominant for all variables with the relative contribution larger than $90 \%$, and $U_{R, 95} \leq 3 \%$ by using $t_{95}=2$ for $v_{R} \geq$ 50.

The present UA estimations for velocity variables are comparable with previous UA's for planar PIV measurements. Gui et al. (2001) observed 2.4\% and Longo et al. (2007) 1.6\% and $1 \sim$ $3.5 \%$ from steady and unsteady flow measurements, respectively.However, the present UA estimations for turbulence variables are larger than the $4 \sim 6 \%$ by Gui et al. (2001) and the $3 \sim 6 \%$ by Longo et al. (2007) for steady flow measurements, however comparable with the $10 \sim 45 \%$ of Longo et al. (2007) for the unsteady flow measurements. For the present PIV, a separate measurement using a Pitot-static tube shows $U / U_{\infty}=0.99$ at the laser plane, where $U_{\infty}$ is the far upstream velocity. Thus, the blockage effect by the underwater camera housings of the present PIV may be attributed in part to the uncertainty in the velocity measurement.The present uncertainty estimates for the turbulence variables are comparable with the aforementioned statistical conver- 
gence error $\left(E_{\mathrm{SC}}^{\mathrm{L}}, E_{\mathrm{SC}}^{\mathrm{U}}\right)$ values, indicating that the random uncertainty may mainly originate from the relatively small number of samples $(N \approx 250)$.

\section{FLOW MEASUREMENT OVERVIEW}

The mean axial velocity $U_{\text {mean }}$ over a flow measurement area $A$ is defined by using a conditional averaging as

$$
U_{\text {mean }}=\int_{A} U \cdot p(U) d A / \int_{A} p(U) d A
$$

In Eq.(14), the conditioning function $p(U)=1$ if $U \leq 0.9$, otherwise $p(U)=0$. Similarly, the mean turbulent kinetic energy $k_{\text {mean }}$ is defined as

$$
k_{\text {mean }}=\int_{A} k \cdot q(k) d A / \int_{A} q(k) d A
$$

where, $q(k)=1$ if $k \geq 0.001$, otherwise $q(k)=0$. Thus, $U_{\text {mean }}$ or $k_{\text {mean }}$ represents approximately the mean $U$ or $k$ within the flow boundary layer. The maximum turbulent kinetic energy $k_{\max }$ may occur close to the hull surface or near the vortex core depending on the phase of the hull motion. Thus, $k_{\max }$ is also defined by using conditional averaging as

$$
k_{\max }=\int_{A} k \cdot r(k) d A / \int_{A} r(k) d A
$$

where, the conditioning function $r(k)=1$ if $k \geq 0.01$, otherwise $r(k)=0$.

The periodic changes of these variables are expressed with a Fourier Series (FS) such as

$$
x(t)=A_{0}+\sum_{n=1} A_{n} \cos \left(2 \pi n f t-\theta_{n}\right)
$$

In Eq.(17), $A_{0}$ is the static component and $A_{n}=\sqrt{a_{n}^{2}+b_{n}^{2}}$ and $\theta_{n}=\arctan \left(b_{n} / a_{n}\right)$ are the $n^{\text {th }}$. order harmonic amplitude and phase, respectively, and $a_{n}$ and $b_{n}$ are the Fourier cosine and sine coefficients of $x(t)$, respectively.

Lastly, the mean Reynolds stress is calculated over the same flow domain as for $k_{\text {mean }}$ but with additional averaging over one PMM cycle as 


$$
\left\langle u_{i} u_{j}\right\rangle=\frac{1}{T} \int_{0}^{T}\left(\int_{A} u_{i} u_{j} \cdot q(k) d A / \int_{A} q(k) d A\right) d t
$$

for the normal stress components $(u u, v v, w w)$, and

$$
\left\langle u_{i} u_{j}\right\rangle_{\mathrm{rms}}=\frac{1}{T} \int_{0}^{T}\left(\sqrt{\int_{A}\left(u_{i} u_{j}\right)^{2} q(k) d A / \int_{A} q(k) d A}\right) d t
$$

for the shear stress components $(u v, u w, v w)$, where $T=1 / f$ is the period of one PMM cycle. The angle-brackets symbol $\langle x\rangle$ is also used for any variable $x$ in the remainder of the present paper to indicate temporal averaging of the variable over one PMM cycle. The conditioning function $q(k)$ in Eqs. (18)and (19)is the same as in Eq. (15). Non-dimensional normal $\left\langle u_{i} u_{j}\right\rangle /\langle 2 k\rangle$ and shear $\left\langle u_{i} u_{j}\right\rangle_{\mathrm{rms}} /\langle 2 k\rangle$ stress valuesare also used in the following discussions, where $\langle 2 k\rangle=\langle u u\rangle+$ $\langle v v\rangle+\langle w w\rangle$.

\subsection{Pure sway flow}

Fig. 3shows the axial velocity $U$, cross-flow $V W$ velocity vectors and turbulent kinetic energy $k$ at $x=0.235$ and 0.935 for $\gamma=0^{\circ}$. The $V W$-vectors are shown using every $8^{\text {th }}$ vector in both $y$ and $z$ directions. The mean axial velocity is nearly constant through the cycle,with $U_{\text {mean }}$ $=0.78 \sim 0.85$. The cross-flow vectors are mainly in the lateral direction caused by the hull translational motion, but locally in rotation due to the presence of vortices. The overall shapes and the peak locations of $k$ exhibit apparent coherences with the velocity-deficit regions. The average turbulence-intensity $\left(I_{\%}=\sqrt{2 k / 3} \times 100\right)$ is about $6 \%$ with $k_{\text {mean }}=0.004 \sim 0.008$. The local turbulence is large inside the boundary layer and near the vortex core, showing $I_{\%} \approx 9$ with $k_{\max }=$ $0.011 \sim 0.015$. The $u u$ component is the largest normal stress with $\left\langle u_{i} u_{j}\right\rangle /\langle 2 k\rangle \approx(0.49,0.35$, $0.16)$ for $(u u, v v, w w)$ and the $u v$ component is the largest shear stress with $\left\langle u_{i} u_{j}\right\rangle_{\mathrm{rms}} /\langle 2 k\rangle \approx$ $(0.25,0.07,0.08)$ for $(u v, u w, v w)$.

\subsection{Pure yaw flow}

Fig. 4 shows the axial velocity $U$, cross-flow $V W$ velocity vectors and turbulent kinetic energy $k$ at $x=0.135,0.535$ and 0.935 for $\gamma=135^{\circ}$. The $U$ contours show a developing boun- 
dary layer structure around the hull. The boundary layer is initially thin at the fore-body locations, then as expected, grows thicker through the aft-body and becomes considerably thicker at the stern; additionally the boundary layer thickness is expanding or contracting in time through the pure yaw motion. Generally, $U_{\text {mean }}=0.72 \sim 0.83$ within the flow. The $V W$-vectors are shown using every $12^{\text {th }}$ vector from the entire measurement grid points in both $y$ and $z$ directions. In general, the velocity vectors are pointing to portside at the fore-body; nearly stagnant around the mid-hull; and then pointing to starboard at the aft-body locations as the hull is turning clockwise viewed from the top. The velocity vectors also clearly show circular fluid motions caused by the presence of the major vortices. The $k$ contours show a coherent structure generally following the shape of the viscous parts of the $U$ contours. The mean turbulence intensity $I_{\%}=4$ $\sim 5$ with $k_{\text {mean }}=0.002 \sim 0.004$. A higher turbulence level occurs where a larger velocity deficit occurs, where the local peak turbulence becomes as high as $I_{\%}=8 \sim 11$ with $k_{\max }=0.011 \sim$ 0.017 .

Fig. 5 shows the changes of $A_{0}$ and $A_{2}$ values of $U_{\text {mean }}, k_{\text {mean }}$, and $k_{\text {max }}$ as functions of $x$ along the hull length. The mean velocity $U_{\text {mean }}$ is the largest at the bow with $A_{0}=0.82$ at $x=$ 0.135 and 0.335 . As the boundary layer grows in size, $A_{0}$ decreases gradually to 0.79 by $x=$ 0.935, approximately in a linear fashion, then drops relatively rapidly to 0.75 at $x=1.035$ as the flow leaves the transom stern. The temporal change in $U_{\text {mean }}$ is moderate with the $A_{2}$ values about $1 \sim 1.5 \%$ of $A_{0}$, but the change becomes relatively larger at $x=1.035$ with $A_{2} \approx 3 \%$ of $A_{0}$. The largest $k_{\text {mean }}$ occurs at $x=1.035$ with $A_{0}=0.0036$ and the second largest at $x=0.135$ with $A_{0}=0.0034$; otherwise the turbulence is nearly stable with $A_{0} \approx 0.003$. The temporal change in $k_{\text {mean }}$ is the largest at $x=0.135$ with $A_{2} \approx 20 \%$ of $A_{0}$ and the smallest at $x=0.335$ with $A_{2} \approx 2 \%$ of $A_{0}$; otherwise $A_{2}=6 \sim 9 \%$ of $A_{0}$. The local peak turbulence $k_{\max }$ decreases gradually from 0.014 at $x=0.135$ to 0.011 at $x=0.935$, then the value increases sharply to 0.014 at $x=1.035$. The temporal change in $k_{\max }$ is the largest at $x=0.135$ with $A_{2} \approx 11 \%$ of $A_{0}$, whereas $A_{2}=2 \sim$ $4 \%$ of $A_{0}$ at other $x$ locations.

The Reynolds normal stress components exhibit anisotropic turbulence with $\left\langle u_{i} u_{j}\right\rangle /\langle 2 k\rangle \approx(0.5,0.3,0.2)$ for $(u u, v v, w w)$, where the axial $u u$ component is the largest. The Reynolds shear stress components usually exhibit an order smaller value with $\left\langle u_{i} u_{j}\right\rangle_{\mathrm{rms}} /\langle 2 k\rangle \approx$ $(0.2,0.08,0.05)$ for $(u v, u w, v w)$, respectively, where the $u v$ component is the largest. 


\section{INTEGRATED CFD AND VORTEX IDENTIFICATION}

\subsection{CFD Simulations}

Computational predictions by Bhushan et al. (2015) show three major vortices shedding from the hull in straight-ahead condition, which are sonar dome vortex (SDV), fore-body keel vortex (FBKV), and bilge keel vortex (BKV). SDV originates from the sonar-dome bow surface, FBKV underneath the fore-body keel, and BKV along the bilge keel of the hull. Since the flow is symmetric about the hull center-plane, these vortices form counter-rotating vortex pairs with one vortex located at the port side and its counter-part vortex at the starboard side. These vortices are rather weak in strength and decay rapidly with increasing $x$ downstream.

Bhushan et al. (2015) also show that the cross-flow induced by drift angle disrupts flow symmetry and enhances the vortex strength significantly when the hull is in static drift condition. The SDV pair merges into a single sonar-dome tip vortex (SDTV) directed leeward. The windward side BKV turns into a bilge-keel tip vortex (BKTV), also convecting toward the leeward side for increasing $x$ downstream. An aft-body keel vortex (ABKV) develops underneath the aftbody keel of the hull. Sakamoto et al. (2012) show similar vortices from steady turning simulations, where the vortices exhibit curved trajectories following the circular path-line of the hull.

Sakamoto et al. (2012) also performed computational simulations for dynamic pure sway and pure yaw maneuvers as shown in Fig. 6. They visualized vortices by using the $Q$-criterion (Hunt et al., 1988) with colored by the normalized helicity density $H_{\mathrm{n}}$ (Levy et al., 1990). When viewed from downstream, a positive $H_{\mathrm{n}}$ (red-colored) signifies counter-clockwise rotation and a negative $H_{\mathrm{n}}$ (blue-colored) signifies clockwise rotation. Sakamoto et al. (2012) used slightly different acronyms of vortices than Bhushan et al. (2015) such as SD for SDV, FK for FBKV, BK for BKV and AK for ABKV. Additionally, Sakamoto et al. (2012) define free surface (FS) vortex and transom (T) vortex, and use a subscript ' $p$ ', 's', or 'c' to indicate the origin of each vortex, representing portside, starboard side, or center, respectively.

Fig. 6(a) shows the pure sway flow at a non-dimensional time $t / T=0$ corresponding to $\gamma$ $=0^{\circ}$, where $T=1 / f$ is the period of one PMM cycle. The hull is translating from the starboard side to the portside, thus the portside is windward and the starboard side is leeward. The flow resembles the static drift flow where $\mathrm{SD}_{\mathrm{S}}$ is the largest vortex. The vortex further stretches in length at $\gamma \approx 45^{\circ}$, becomes thinner and more straightened at $\gamma \approx 90^{\circ}$, and then detaches from the sonar dome surface and $\mathrm{SD}_{\mathrm{P}}$ begins to form at $\gamma \approx 135^{\circ}$. The $\mathrm{BK}_{\mathrm{P}}$ and $\mathrm{BK}_{\mathrm{S}}$ are the second largest 
vortices and exhibit similar dynamic behaviors as $\mathrm{SD}_{\mathrm{S}}$, i.e., stretching, straightening, thinning, and then changing its direction of rotation.

Fig. 6(b) shows pure yaw flow at $t / T=0.375$ (or $\gamma=135^{\circ}$ ). The hull is in a clockwise turning motion when viewed from the top, thus the bow is directing toward the starboard side and the stern is directing toward the portside. The flow resembles the steady turning flow where $\mathrm{SD}_{\mathrm{P}}$ is the largest vortex. Initially, $\mathrm{SD}_{\mathrm{S}}$ is the major vortex at $\gamma \approx 0^{\circ}$, then the vortex detaches from the sonar dome surface at $\gamma \approx 45^{\circ}$ and its counter-part vortex $\mathrm{SD}_{\mathrm{P}}$ begins to form, then the vortex grows and becomes the major vortex by $\gamma \approx 135^{\circ} . \mathrm{BK}_{\mathrm{P}, \mathrm{S}}$ and $\mathrm{AK}_{\mathrm{C}}$ are the second largest vortices, whereas $\mathrm{FK}_{\mathrm{P}, \mathrm{S}}$ are relatively small.

\subsection{Vortex Identification}

Fig. 7 shows the major vortices observed from the present experimental data for pure sway and pure yaw maneuvers. The vortices are visualized by using the iso-contours of axial vorticity

$$
\omega_{x}=\left(\frac{\partial W}{\partial y}-\frac{\partial V}{\partial z}\right)
$$

The red colored contours signify positive $\omega_{x}$ (counter-clockwise rotation) and the blue colored contours signify negative $\omega_{x}$ (clockwise rotation). The sign and location of local peak $\omega_{x}$ are cross-referenced with the $Q$ iso-surface and $H_{\mathrm{n}}$ from the CFD to identify each vortex. The vortex naming in this section follows Sakamoto et al. (2012) for direct comparisons. However, the remainder of the present study will use the acronyms by Bhushan et al. (2015). Additionally, the vortex naming in the present study will use a subscript 'P' or ' $\mathrm{S}$ ' for 'Portside' or 'Starboard', respectively, similarly as in Sakamoto et al. (2012).

Fig. 7(a) shows the $\omega_{x}$ contours for pure sway at $\gamma=0^{\circ}$. The $\mathrm{SD}_{\mathrm{S}}$ is seen at $x=0.135$ and 0.225 , with displaced position leeward downstream of the sonar dome bow. However, the $\mathrm{SD}_{\mathrm{S}}$ is out of the field of view of the present measurement at the aft-body locations thus not seen either at $x=0.735$ or 0.935 . The present measurement also misses a part of the data for $\mathrm{SD}_{\mathrm{S}}$ at $x=$ 0.135 (marked with a dashed-circle in the insertion box). However, the sign and location of the nearby $\omega_{x}$ contour provide an indication of the presence of the vortex and its identity. A pair of counter-rotating secondary vortices, each named as $\mathrm{A}$ and $\mathrm{B}$, is present next to the $\mathrm{SD}_{\mathrm{S}}$ at $x=$ 
0.135. The identity of these secondary vortices will be discussed together with those found from the pure yaw flow. The $\mathrm{FK}_{\mathrm{P}}$ is seen at $x=0.235$ but the $\mathrm{FK}_{\mathrm{S}}$ is not well resolved, possibly due to the lack in proximity of the present measurement close to the hull surface. The $\mathrm{BK}_{\mathrm{P}}$ and $\mathrm{BK}_{\mathrm{S}}$ are seen at $x=0.735$ and 0.935 , both displaced leeward as predicted by CFD. The $\mathrm{AK}_{\mathrm{C}}$ is resolved only at $x=0.935$.

Fig. 7(b) shows the $\omega_{x}$ contours for pure yaw at $\gamma=135^{\circ}$. The clockwise rotating $\mathrm{SD}_{\mathrm{P}}$ is well resolved through the all $x$ measurement locations. The counter-rotating $\mathrm{FK}_{\mathrm{P}}$ and $\mathrm{FK}_{\mathrm{S}}$ pair is seen between $x=0.135$ and 0.535 . However, the $\mathrm{FK}_{\mathrm{S}}$ is not seen clearly from further downstream locations. The positive $\omega_{x}$ contours located between the $\mathrm{SD}_{\mathrm{P}}$ and $\mathrm{BK}_{\mathrm{S}}$ at the aft-body locations seem to be the persistence of $\mathrm{FK}_{\mathrm{P}}$ with increasing $x$ downstream. However, the CFD prediction in Fig. 3(b) does not agree with this observation and the identity of those $\omega_{x}$ contours is unclear. The clockwise rotating $\mathrm{BK}_{\mathrm{P}}$ and $\mathrm{BK}_{\mathrm{S}}$ are resolved between $x=0.535$ and 1.035. The $\mathrm{AK}_{\mathrm{C}}$ is also in clockwise rotation and seen from $x=0.935$ and 1.035 .

Fig. 8 shows the close-up views of pure yaw $\omega_{x}$ contours at $x=0.135$. Apparently, three secondary-vortices are observed, each named as A, B and C. Initially, the vortices A and B form with the onset of $\mathrm{SD}_{\mathrm{P}}$. The vortex A grows and the vortex $\mathrm{B}$ weakens while the $\mathrm{SD}_{\mathrm{P}}$ grows. The vortex $\mathrm{C}$ forms as the $\mathrm{SD}_{\mathrm{P}}$ reaches its peak strength. Afterwards, the vortex $\mathrm{C}$ decays first, then the vortices $\mathrm{A}$ and $\mathrm{B}$ weaken and decay with the dissipating SD. Next, these secondary vortices reappear at the portside now with $\mathrm{SD}_{\mathrm{S}}$ and repeat the same process with their signs changed. Sakamoto et al. (2012) detect a secondary vortex formed near the sonar dome surface from their static drift simulations by inspecting the limiting streamlines and identify the vortex as $\mathrm{SD}_{\mathrm{S} 2}$. Bhushan et al. (2015) also observe a secondary vortex formed next to the SDTV from static drift simulations and name the vortex as the sonar-dome bubble vortex (SDBV). Both $\mathrm{SD}_{\mathrm{S} 2}$ and SDBV only appear over a short distance and decay immediately downstream of the sonar dome. The identitiesof these secondary vortices A, B, or C in pure sway or pure yaw flows are considered as the dynamic versions of the $\mathrm{SD}_{\mathrm{S} 2}$ or the $\mathrm{SDBV}$.

Fig. 9 shows the major vortices of pure yaw at $x=0.535$ for $\gamma=$ (a) $0^{\circ}$, (b) $45^{\circ}$, (c) $90^{\circ}$ and (d) $135^{\circ}$. At $\gamma=0^{\circ}$, the $\mathrm{SDV}_{\mathrm{P}}$ is located near the hull center-plane and the $\mathrm{FBKV}_{\mathrm{P}}$ and $\mathrm{FBKV}_{\mathrm{S}}$ pair at the portside underneath the hull bottom surface. The $\mathrm{SDV}_{\mathrm{P}}$ traverses to portside by $\gamma=45^{\circ}$ and dissipates while its counter vortex $\mathrm{SDV}_{\mathrm{S}}$ begins. Then, $\mathrm{SDV}_{\mathrm{S}}$ further grows through the later phases and travels toward the starboard. Both $F B K V_{P}$ and $F B K V_{S}$ traverse to 
starboard across the hull center-plane, with evolutions in their apparent size and strength with increasing phase angle. The $\mathrm{BKV}_{\mathrm{P}}$ and $\mathrm{BKV}_{\mathrm{S}}$ are almost fixed at the tips of the portside and starboard bilge keels, respectively, through the phase change. The relative size of these vortices is noticeably smaller than the other vortices.

Fig. 10 shows the major vortices of pure yaw at $x=0.935$ for the same phases as in Fig. 9. The SDV exhibits a similar behavior as at $x=0.535$, but its transition from $\mathrm{SDV}_{\mathrm{P}}$ to $\mathrm{SDV}_{\mathrm{S}}$ occurs at a slightly later phase, between $\gamma=45^{\circ}$ and $90^{\circ}$. The $\mathrm{BKV}_{\mathrm{P}}$ and $\mathrm{BKV}_{\mathrm{S}}$ exhibit considerably enlarged sizebut seem dispersed and dissipated compared to those at $x=0.535$. The ABKV is located immediately underneath the hull keel line, traversing from the portside to starboard. The ABKV changes its sign from negative to positive by $\gamma=45^{\circ}$. The FBKV pair is not observed at this $x$ location. However, as discussed earlier with Fig. 7(b), it is not clearly understood if the identity of the vortex located between the $B K V_{P}$ and $A B K V$ at $\gamma=0^{\circ}$ or the vortex between the $\mathrm{ABKV}$ and $\mathrm{BKV}_{\mathrm{S}}$ at $\gamma=135^{\circ}$ are the extension of the $\mathrm{FBKV}_{\mathrm{S}}$ or $\mathrm{FBKV}_{\mathrm{P}}$, respectively, from the present measurement due to its coarse resolution in the $x$-direction.

\section{VORTEX CORE ANALYSIS}

\subsection{Core Axial Vorticity}

A vortex core is defined herein as the location where the local peak $\omega_{x}$ occurs. Fig. 11(a)(d) show the time-histories of the axial vorticity at the core location $\omega_{x \text {,core }}$ for SDV, FBKV, $\mathrm{BKV}_{\mathrm{S}}$, and $\mathrm{ABKV}$ in the pure yaw maneuver, respectively. The time-histories are reconstructed by using the FS series in Eq. (17) and the $1^{\text {st }}$-order harmonic amplitude $A_{1}$ and phase $\theta_{1}$ are presented in Fig. 12.

SDV exhibits mainly the $1^{\text {st }}$-order harmonic oscillations and the largest $\omega_{x, \text { core }}$ at $x=$ 0.135 with $A_{1}=269$. Subsequently, the $A_{1}$ value decreases quickly and levels out at about 160 between $x=0.335$ and 0.735 , then mildly decreases to 110 by $x=1.035$. The $\theta_{1}$ value increases from $-75^{\circ}$ at $x=0.135$ to $16^{\circ}$ at $x=1.035$, almost linearly as $\theta_{1}(x) \approx k x-\pi / 2$ in radians, where the slope $k=5 \pi / 9$. This suggests that $\omega_{x \text {,core }}$ of SDV propagates in space and time in a wavelike form such that $\omega_{x, \text { core }}(x, t)=A_{1}(x) \sin (k x-\omega t)$ with a phase velocity $v_{p}=\omega / k=18 f / 5$ $=0.96 U_{C}$, where $\omega=2 \pi f$. FBKV $V_{\mathrm{P}}$ exhibits almost constant $\omega_{x, \text { core }}(\approx 40)$ at $x=0.135$ during the $1^{\text {st }}$-half cycle then the $\omega_{x, \text { core }}$ valueincreasesrapidly for the $2^{\text {nd }}$-half cycle with the peak 
$\omega_{x, \text { core }} \approx 200 . \mathrm{FBKV}_{\mathrm{P}}$ at $x=0.335$ or 0.535 shows almost constant $\omega_{x, \text { core }}(\approx 33$ and 20 , respectively) through the whole cycle. The trend for $\mathrm{FBKV}_{\mathrm{S}}$ is the antisymmetric mirrored-images of the trend for $\mathrm{FBKV}_{\mathrm{P}}$. BKV $\mathrm{V}_{\mathrm{S}}$ exhibits a sinusoidal undulation in $\omega_{x \text {,core }}$ which is superimposed on a steady value. The steady value is usually small, about -14 and -7 at $x=0.535$ and 0.735 , respectively and nearly zero at the remaining $x$ locations. The $1^{\text {st }}$-order harmonic amplitude is approximately linear with $x$ such that $A_{1} \approx-125 x+150$ and the $1^{\text {st }}$-order phase angle is nearly constant with $\theta_{1} \approx-60^{\circ}$ for all $x$ locations except for $x=0.735$ where $\theta_{1}=-15^{\circ}$. The reason for this offtrend in $\theta_{1}$ at $x=0.735$ is not clearly understood from the present study. ABKV also exhibits a

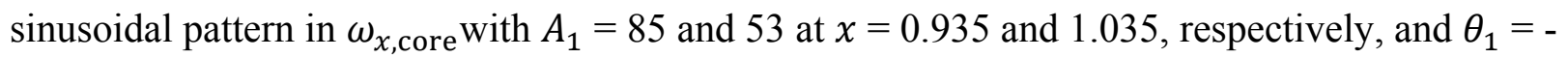
$33^{\circ}$ and $-60^{\circ}$, respectively.

\subsection{Core Trajectories}

The transverse and vertical locations of the vortex cores, $y_{\text {core }}$ and $z_{\text {core }}$, respectively, are traced to build vortex core trajectories in the cross plane. The core trajectories of SDV, FBKV $\mathrm{S}_{\mathrm{S}}$ $\mathrm{BKV}_{\mathrm{S}}$ and $\mathrm{ABKV}$ are shown in Figs. 13-16. Typically, the trajectories appear as a closed-loop shape. The width of the trajectoryis defined as the transverse extent of the loop from side to side, or $\mathrm{W}_{\text {trj }}=\max \left(y_{\text {core }}\right)-\min \left(y_{\text {core }}\right)$ and the height of the trajectoryas the vertical extent of the loop from base to top, $\operatorname{orH}_{\mathrm{trj}}=\max \left(z_{\text {core }}\right)-\min \left(z_{\text {core }}\right)$. In addition, the average values of $y_{\text {core }}$ and $z_{\text {core }}$ over one PMM cycle are used to define the mean-displacement and -depth of the trajectory, $\left\langle y_{\text {core }}\right\rangle$ and $\left\langle z_{\text {core }}\right\rangle$, respectively. Fig. 17 shows the $\mathrm{W}_{\text {trj }}$ and $\mathrm{H}_{\text {trj }}$ values at all $x$ measurement locations and Fig. 18 shows the $\left\langle y_{\text {core }}\right\rangle$ and $\left\langle z_{\text {core }}\right\rangle$ values of each vortex trajectory.

Fig. 13shows theSDV core trajectories. Initially, the trajectory forms two arc-shaped loops at $x=0.135$,one by theSDV $\mathrm{P}$ and the other one by theSDV $\mathrm{S}_{\mathrm{S}}$ The $\mathrm{SDV}_{\mathrm{P}}$ trajectory begins to form fromthe port side,traverses to the starboard side, and then returns to the portside. The $\mathrm{SDV}_{\mathrm{S}}$ trajectory follows and repeats this process but in a mirrored way, i.e., begins from the starboard side, traverses to the portside, and then returns to the starboard side. The trajectory at further downstream $x$ locations exhibits a pair of two bow-tie-shaped loops at $x=0.335$, a single bow-tie shape at $x=0.535$, a pair of two hook-shaped loops at $x=0.735$, andfigure-eight-shaped loops at $x=0.935$ and 1.035 . The height and width of the trajectory at $x=0.135$ is small with $\mathrm{W}_{\text {trj }}=0.08 \mathrm{~B}_{\mathrm{WL}}$ and $\mathrm{H}_{\mathrm{trj}}=0.09 \mathrm{~T}_{\mathrm{m}}$. The width grows to $0.27 \mathrm{~B}_{\mathrm{WL}}$ at $x=0.335$ then slightly contracts to $0.22 \mathrm{~B}_{\mathrm{WL}}$ at $x=0.535$. Then, the width continues growing with increasing downstream $x$ 
locations, up to $0.75 \mathrm{~B}_{\mathrm{WL}}$ at $x=1.035$, approximately in a quadratic manner. The height grows approximately linearly with $x$, up to $\mathrm{H}_{\mathrm{trj}}=0.52 \mathrm{~T}_{\mathrm{m}}$ at $x=1.035$. The mean depth of the trajectory is nearly constant at about $1.3 \sim 1.4 \mathrm{~T}_{\mathrm{m}}$ between $x=0.135$ and 0.735 . Then, the mean depth further downstream decreases rapidly to $0.75 \mathrm{~T}_{\mathrm{m}}$ by $x=1.035$.

Fig. 14 shows the core trajectories of $\mathrm{FBKV}_{\mathrm{P}}$ and $\mathrm{FBKV}_{\mathrm{S}}$. The trajectory for FBKV $\mathrm{V}_{\mathrm{P}}$ at $x$ $=0.135$ initially makes an orbit-like double-loop then forms a single-loop which isextruded toward the hull center-plane. Whereas the counterpart FBKV $\mathrm{F}_{\mathrm{S}}$ makes the extruded single-loop first then the orbit-like double-loop trajectory. The occurrence of the extruded loop coincides with the peak $\omega_{x, \text { core }}$ of SDV and FBKV as shown in Fig. 11(a) and (b), respectively. For the case of FBK $V_{P}$, as can be seen from Fig. 8, the nearby passing $S_{D V}$ with its peak $\omega_{x \text {,core }}$ may reinforce and attract the co-rotating $\mathrm{FBKV}_{\mathrm{P}}$ to form the extruded loop trajectory. However, the size of the trajectory is very small with only $\mathrm{W}_{\mathrm{trj}}=0.01 \mathrm{~B}_{\mathrm{WL}}$ and $\mathrm{H}_{\mathrm{trj}}=0.03 \mathrm{~T}_{\mathrm{m}}$. The trajectory at downstream locations widens with $\mathrm{W}_{\mathrm{trj}}=0.18$ and $0.33 \mathrm{~B}_{\mathrm{WL}}$ at $x=0.335$ and 0.535 , respectively, in an approximately linear manner. The height of trajectory is relatively small with $\mathrm{H}_{\mathrm{trj}}=0.14$ and 0.07 $\mathrm{T}_{\mathrm{m}}$ at $x=0.335$ and 0.535 , respectively, thus the vortex makes almost a purely lateral motion by $x=0.535$. The mean displacement of the trajectory is also small $<0.05 \mathrm{~B}_{\mathrm{WL}}$ and the mean depth is about $1.1 \mathrm{~T}_{\mathrm{m}}$, thus the vortex remains around the hull center-plane and close to the keel.

Fig. 15 shows the $\mathrm{BKV}_{\mathrm{S}}$ core trajectories. The trajectory at $x=0.535$ forms an arcshaped loop that wraps around the bilge keel tip. Then, the trajectories at the later $x$ locations form a wing-section shaped loop with the leading edge part pointing toward the hull centerplane. The width of the loop is small at $x=0.535$ with $\mathrm{W}_{\mathrm{trj}}=0.04 \mathrm{~B}_{\mathrm{WL}}$, and then grows rapidly, with $\mathrm{W}_{\mathrm{trj}}=0.09,0.30$, and 0.43 of $\mathrm{B}_{\mathrm{WL}}$ at $x=0.735,0.935$ and 1.035 , respectively. The height of the loop also grows rapidly with $\mathrm{H}_{\mathrm{trj}}=0.07,0.16,0.40$ and $0.52 \mathrm{~T}_{\mathrm{m}}$, respectively. With this fast growth in trajectory size, the vortex also diffuses and weakens quickly with increasing $x$ as can be seen from Fig. 7. The mean displacement of the trajectory is $0.38,0.36,0.24$, and $0.20 \mathrm{~B}_{\mathrm{WL}}$ and the mean depth is $0.97,0.78,0.38$, and $0.19 \mathrm{~T}_{\mathrm{m}}$, at $x=0.535,0.735,0.935$, and 1.035 , respectively.

Fig. 16 shows the ABKV core trajectories. The trajectories form a figure-eight-shaped loop. At $x=0.935$, the width and height of the trajectory are $\mathrm{W}_{\mathrm{trj}}=0.16 \mathrm{~B}_{\mathrm{WL}}$ and $\mathrm{H}_{\mathrm{trj}}=0.08 \mathrm{~T}_{\mathrm{m}}$. The trajectory at $x=1.035$ appears as a nearly translational motion in the horizontal plane with 
$\mathrm{W}_{\text {trj }}=0.23 \mathrm{~B}_{\mathrm{WL}}$ and $\mathrm{H}_{\mathrm{trj}}=0.10 \mathrm{~T}_{\mathrm{m}}$. The vortex locates around the hull center-plane and close to the bottom surface with the mean depth 0.46 and $0.11 \mathrm{~T}_{\mathrm{m}}$ at $x=0.935$ and 1.035 , respectively.

\subsection{Core Variables}

The core axial $U_{\text {core }}$, transverse $V_{\text {core }}$, and vertical $W_{\text {core }}$ velocity components and the core turbulent kinetic energy $k_{\text {core }}$ values are also traced. Fig. 19 presents the average values of these variablesover one complete cycle of pure yaw, $\left\langle U_{\text {core }}\right\rangle,\left\langle V_{\text {core }}\right\rangle_{\text {rms }},\left\langle W_{\text {core }}\right\rangle$, and $\left\langle k_{\text {core }}\right\rangle$, and thepeak- $k_{\text {core }}$ values as functions of $x$ for each major vortex. Usually, these variables exhibit mild changes with $x$, thus the discussions herein focus on their general trends by further averaging along $x$.For any variable $x$, an over-bar symbol, e.g. $\bar{x}$, indicates averaging along the $x$-axis.

The mean axial core velocity $\left\langle U_{\text {core }}\right\rangle=0.66 \sim 0.92$ in general over all vortex systems. SDV exhibits the largest $\overline{\left\langle U_{\text {core }}\right\rangle}=0.84$ followed by BKV and FBKV with $\overline{\left\langle U_{\text {core }}\right\rangle}=0.83$ and 0.78 , respectively, and ABKV shows the smallest $\overline{\left\langle U_{\text {core }}\right\rangle}=0.67$. Usually, $\left\langle V_{\text {core }}\right\rangle_{\mathrm{rms}}=0.03 \sim$ 0.12 for all vortices, where SDV shows the largest $\overline{\left\langle V_{\text {core }}\right\rangle_{\mathrm{rms}}}=0.08$ and ABKV the smallest $\overline{\left\langle V_{\text {core }}\right\rangle_{\mathrm{rms}}}=0.04 .\left\langle W_{\text {core }}\right\rangle=-0.05 \sim 0.10$ and $\mathrm{ABKV}$ shows the largest $\overline{\left\langle W_{\text {core }}\right\rangle}=0.09$ and FBKV the smallest $\overline{\left\langle W_{\text {core }}\right\rangle}=-0.03$. The mean core turbulence $\left\langle k_{\text {core }}\right\rangle=0.002 \sim 0.007$ corresponding to mean turbulence intensity of $4 \sim 7 \%$. The mean turbulence is the largest for SDV and ABKV with $\overline{\left\langle k_{\text {core }}\right\rangle}=0.005$. SDV also exhibits the largest local peak- $k_{\text {core }}=0.013$ among other vortices. BKV exhibits the smallest local peak- $k_{\text {core }}=0.004$.

\section{CONCLUSIONS AND FUTURE WORK}

Towing tank experiments for a surface combatant in dynamic pure sway and pure yaw maneuvers are presented. The data include phase-averaged velocities, turbulentkinetic energy, axial vorticity fields and uncertainty assessment. The geometry is a 1/46.6 scale DTMB model 5512 of $L=3.048 \mathrm{~m}$. DTMB model 5512 is a geosim of DTMB 5415 model. The experiments are performed in a $100 \mathrm{~m} \times 3 \times \mathrm{m} \times 3 \mathrm{~m}$ towing tank. The measurement system is a customdesigned towing-tank maneuvering test flow-mapping system, which features a planar motion mechanism for captive model testing with an integrated SPIV.

The quality of phase-averaged data is assessed by monitoring the statistical convergence error and following standard uncertainty assessment procedures. Statistical convergence error is usually less than $1 \%$ of the towing speed $U_{C}$ for the velocity data and less than $20 \%$ of the range 
value of turbulent kinetic energy, $[k]$, for the Reynolds stress components. The error is larger locally where the flow turbulence is higher. The uncertainty is assessed as $2 \sim 3 \%$ of $U_{C}$ for the velocity variables and $10 \sim 35 \%$ of $[k]$ for the turbulence variables, inside the flow boundary layer. The uncertainty is smaller atfar-field locations outside the flow boundary layer, typically less than $1 \%$ and $5 \%$ for velocity and turbulence variables, respectively.

The identification of flow vortices is done by cross-referencing the experimental data to the integrated computational simulation results. The computation predicts four major vortices, which are sonar dome vortex, fore-body keel vortex, bilge keel vortex, and aft-body keel vortex. The apparent locations and the directions of rotation agree well between the computational prediction and the experiment for these major vortices. However, sparse data from the present measurements in the $x$-coordinate do not provide sufficient information to identify minor vortices. The flow-field results indicate maneuvering-induced vortices and their interaction with the turbulent boundary layer. The core locations and trajectories of the major vortices are traced and the flow variables at the core locations are analyzed.

The present work is important for establishing a readily accessible maneuvering dataset with documentation of the test conditions and rigorous and comprehensive uncertainty assessment of the measurement results.A preliminary publication of the present work (Longo et al., 2006) has been recognized by the $26^{\text {th }}$ ITTC Special Committee on Detailed Flow Measurements as the first application of towed PIV for the study of a maneuvering ship. The results are also useful for CFD validation and are archived at http://www.iihr.uiowa.edu/shiphydro. The present data (Part I and Part II together) are the benchmark case of the SIMMAN (Workshop on Verification and Validation of Ship Manoeuvring Simulation Method) 2008 and the SIMMAN 2014 as well. The global forces, moment, and motion data and local flow field data are compared with the computational simulation results using various numerical schemes, turbulence models, and computational grid sizes. Future work will focus ontomographic PIV flow measurements for a fully appended model in dynamic maneuvers, under the auspices of NATO AVT-253-RTG Assessment of Prediction Methods for Large Amplitude Dynamic Maneuvers for Naval Vehicles. The AVT-253-RTG builds on the NATO AVT-183 Reliable Predictions of Separated Flow Onset and Progression for Air and Sea Vehicles that focused on large-amplitude static drift maneuvers. 


\section{ACKNOWLEDGMENTS}

This research was sponsored by the Office of Naval Research under Grant N00014-10-10017 under the administration of Dr. Ki-Han Kim and Dr. Thomas Fu whose support is greatly appreciated. The authors would like to express thanks to IIHRs electronics supervisor Doug Houser who designed, built, and oversaw testing of the PIV synchronizer. Without his hard work and expertise, this study would not have been possible. Special thanks are also extended to University of Iowa mechanical and civil engineering undergraduates (Evan Aschbrenner, Peter Carusona, Mike Elgin, Nathan Hicks, Matt Marquardt, Ryan Nielsen, Rob White, Issac Johnson, Scott Salsbery, and Chelsea Cross) who assisted in the data acquisition phase of this study.

\section{REFERENCES}

Abdel-Maksoud, M., Müller, V., Xing, T.,Toxopeus, S.,Stern, F., Kim, S., Shawn, A.,Petterson, K.,Tormalm, M.,Gietz, U., Schiller, P., Rung, T., 2015, "Experimental and Numerical Investigations on Flow Characteristics of the KVLCC2 at $30^{\circ}$ Drift Angle," proposed abstract $5^{\text {th }}$ World Maritime Technology Conference, Providence, Rhode Island, USA.

ASME, 1998, Test Uncertainty: Instruments and Apparatus, PTC 19.1-1998.

ASME, 2005, TestUncertainty, PTC 19.1-2005.

Bhushan, S., Yoon, H., Stern, F., Guilmiuau, E., Visonneau, M., Toxopeus, S., Simonsen, C., Aram, S., Kim, S-E, Griporopoulos, G., Petterson, K., and Fureby, C., 2015, "CFD Validation for Surface Combatant 5415 Straight Ahead and Static Drift 20 Degree Conditions," Chapter 4, NATO Report CSO-AVT-183 (in preparation).

Broglia, R., Zaghi, S., Campana, E.F., Visonneau, M., Queutey, P., Dogan, T. Sadat-Hosseini, H., Stern, F., and Milanov, E., 2015, “CFD Validation for Delft 372 Catamaran in Static Drift Conditions, Including Onset and Progression Analysis," proposed abstract $5^{\text {th }}$ World Maritime Technology Conference, Providence, Rhode Island, USA.

Gui, L., Longo, J., and Stern, F., 2001, "Towing tank PIV measurement system, data and uncertainty assessment for DTMB model 5512,” Experiments in Fluids, 31, 336 - 346.

Hunt, J. C. R., Wray, A. A., and Moin, P., 1988, "Eddies, Stream and Convergence Zones in Turbulent Flows," Center for Turbulence Research Report CTR-S88, pp. 193-208.

Irvine, M., Longo, J., and Stern, F., 2013, "Forward Speed Calm Water Roll Decay for Surface Combatant 5415: Global and Local Flow Measurements," Journal of Ship Research, Vol. 57, No. 4, pp. 202-219.

ISO, 1995, Guide to the Expression of Uncertainty in Measurement, Geneva: International Organization for Standardization. 
ITTC, 2002a, Report of the Resistance and Flow Committee, Proceedings, $23^{\text {rd }}$ International Towing Tanks Conference, Venice, Italy.

ITTC, 2002b, Quality Manual, $23^{\text {rd }}$ International Towing Tank Conference, Venice, Italy.

ITTC, 2011, Proceedings, $26^{\text {th }}$ International Towing Tank Conference, Rio de Janeiro, Brazil.

ITTC, 2014, Proceedings, $27^{\text {th }}$ International Towing Tank Conference, Copenhagen, Denmark.

Larsson, L., Stern, F., and Visonneau, M. (editors), 2014,Numerical Ship Hydrodynamics: An Assessment of the Gothenburg 2010 Workshop, Springer.

Levy et al. 1990, Y., Degani, D., and Seigner, A., 1990, "Graphical Visualization of Vortical Flows by Means of Helicity," AIAA Journal, 28(8), pp. 1347-1352.

Longo, J. Gui, L., and Stern, F., 2004, "Ship Velocity Fields," PIV and Water Waves, Advances in Coastal and Ocean Engineering, World Scientific, Singapore.

Longo, J., Yoon, H.-S., Toda, Y., and Stern F., 2006, "Phase-Average 3DPIV/Wave Elevation and Force/moment Measurements for Surface Combatant in PMM Maneuvers," $26^{\text {th }}$ Symposium on Naval Hydrodynamics, Rome, Italy.

Longo, J., Shao, J., Irvine, M., and Stern, F., 2007, "Phase-Averaged PIV for the Nominal Wake of a Surface Ship in Regular Head Waves," Transactions of the ASME, Vol. 129, 524 - 540.

Metcalf, B., Longo, J., Ghosh, S., and Stern, F., 2006, "Unsteady free-surface wave-induced boundary-layer separation for a surface-piercing NACA 0024 foil: Towing tank experiments," Journal of Fluids and Structures, 22, 77-98.

Sakamoto, N., Carrica, M. P., and Stern, F., 2012, "URANS simulations of static and dynamic maneuvering for surface combatant: Part 2. Analysis and validation for local flow characteristics," Journal of Marine Science and Technology, 17:446-468.

Stern, F. and Agdrup, K. (editors), 2009, "Proceedings of the Workshop on Verification and Validation of Ship Manoeuvring Simulation Methods SIMMAN 2008," FORCE Technology, Sept. 2009, Lyngby, Denmark.

Yoon, H., Gui, L., Bhushan, S., and Stern, F., 2014, "Tomographic PIV Measurements for a Surface Combatant at Straight Ahead and Static Drift Conditions," $30^{\text {th }}$ Symposium on Naval Hydrodynamics, Hobart, Tasmania, Australia.

Yoon, H., Simonsen, C., D., Benedetti, L., Longo, J., Toda, Y., and Stern, F., 2015, "Benchmark CFD Validation Data for Surface Combatant 5415 in PMM Maneuvers - Part I: Force/Moment/Motion Measurements," Ocean Engineering (2015),http://dx.doi.org/10.1016/j.oceaneng.2015.04.087. 
Table 1. UA Summary (Inner region).

\begin{tabular}{ccccccc}
\hline & $\begin{array}{c}\text { Systematic } \\
\text { standard } \\
\text { Variable } \\
\text { uncertainty }\end{array}$ & $\begin{array}{c}\text { Random } \\
\text { standard } \\
\text { uncertainty }\end{array}$ & $\begin{array}{c}\text { Combined } \\
\text { standard } \\
\text { uncertainty }\end{array}$ & $\begin{array}{c}\text { Relative } \\
\text { systematic } \\
\text { uncertainty } \\
\text { contribution }\end{array}$ & $\begin{array}{c}\text { Relative } \\
\text { random } \\
\text { uncertainty } \\
\text { contribution }\end{array}$ & $\begin{array}{c}\text { Expanded } \\
\text { uncertainty }\end{array}$ \\
$(-)$ & $b_{R}$ & $S_{R}$ & $u_{R}$ & $b_{R}^{2} / u_{R}^{2}$ & $s_{R}^{2} / u_{R}^{2}$ & $U_{R, 95}$ \\
\hline$U$ & $(\%)^{1)}$ & $(\%)^{1)}$ & $(\%)^{1)}$ & $(\%)$ & $(\%)$ & $(\%)^{1)}$ \\
$V$ & 0.8 & 1.2 & 1.5 & 29 & 71 & 3.2 \\
$W$ & 1.0 & 0.5 & 1.2 & 77 & 23 & 2.5 \\
$u u$ & 0.8 & 0.6 & 1.1 & 63 & 37 & 2.4 \\
$v v$ & 1.4 & 5.0 & 5.4 & 8 & 92 & 23.2 \\
$w w$ & 0.8 & 2.4 & 2.6 & 10 & 90 & 11.3 \\
$u v$ & 0.3 & 1.4 & 1.4 & 5 & 95 & 6.2 \\
$u w$ & 0.8 & 2.4 & 2.6 & 10 & 90 & 11.3 \\
$v w$ & 0.1 & 1.3 & 1.3 & 1 & 99 & 5.6 \\
$k$ & 0.1 & 0.8 & 0.9 & 2 & 98 & 3.7 \\
\hline & 1.2 & 3.5 & 3.9 & 11 & 89 & 16.8 \\
\hline
\end{tabular}

${ }^{1)}$ Percent of $[k]=0.015$ for $R=u u, v v, w w, u v, u w, v w$ and $k$, where $[k]$ is the range of $k$. 


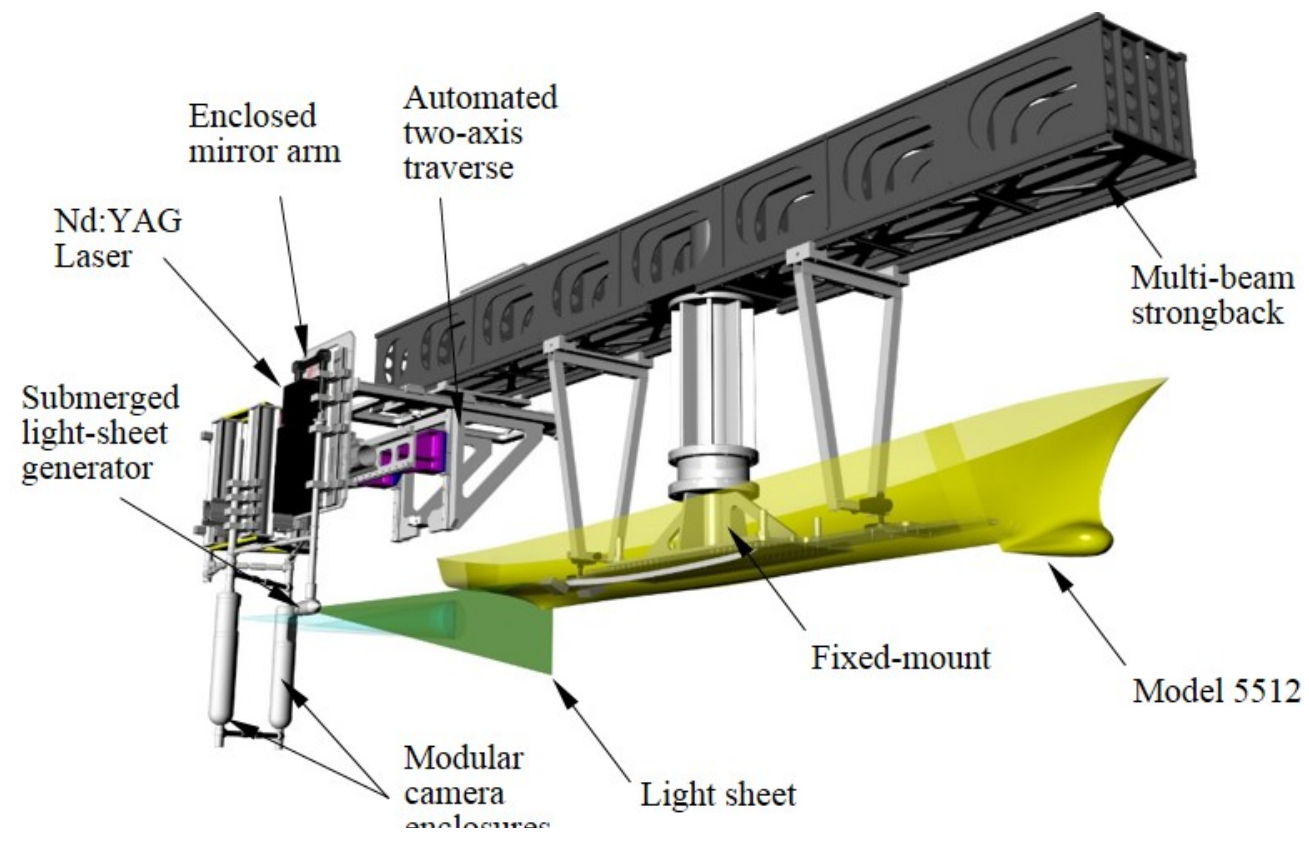

Fig. 1.SPIV setup with model 5512. 
(a)

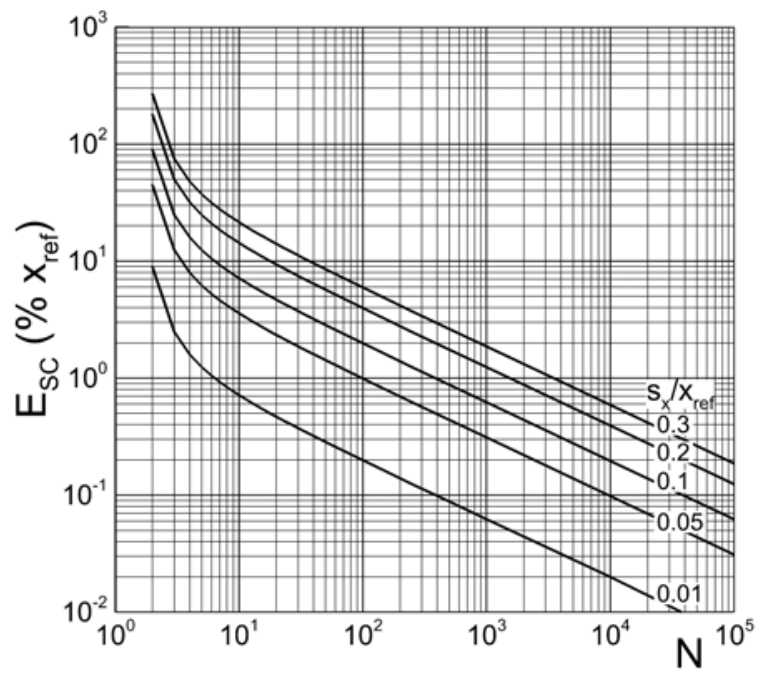

(b)

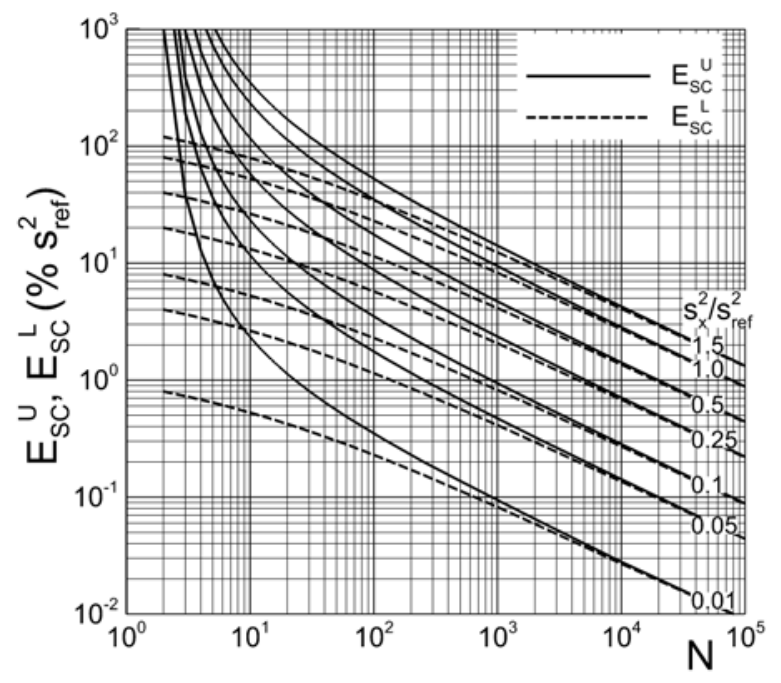

Fig. 2.Statistical convergence errors in (a) mean $\bar{x}$ and (b) variance $s_{x}^{2}$ for a $95 \%$ level of confidence. For a measurement of mean velocity $U(=\bar{x})$, the parameter $s_{x} / x_{\text {ref }}$ corresponds to the flow turbulence intensity $I=u / U$ since $s_{x}$ corresponds to the root-mean-square velocity fluctuation $u$ and by selecting $U$ as $x_{\text {ref. }}$ Thus, for a flow of $I=0.1$ and a sample size $N=100, E_{\mathrm{SC}} \approx 2 \%$ is read from the chart (a) using the $s_{x} / x_{\text {ref }}=0.1$ line. If $E_{\mathrm{SC}} \leq 1 \%$ is desired, $N \geq 400$ is required. If isotropic turbulence is assumed for the same flow, the parameter $s_{x}^{2} / s_{\text {ref }}^{2}=1$ since $s_{x}^{2}=u u$ and if $2 k / 3=u u$ is selected for $s_{\text {ref }}^{2}$. Thus, for the Reynolds normal stress $u u,\left(E_{\mathrm{SC}}^{\mathrm{L}}, E_{\mathrm{SC}}^{\mathrm{U}}\right) \approx(23 \%, 34 \%)$ are read from the chart (b) if sample size $N=100$. If the errors are desired less than $1 \%$, then $N \geq 76,000$ is required. 
(a)
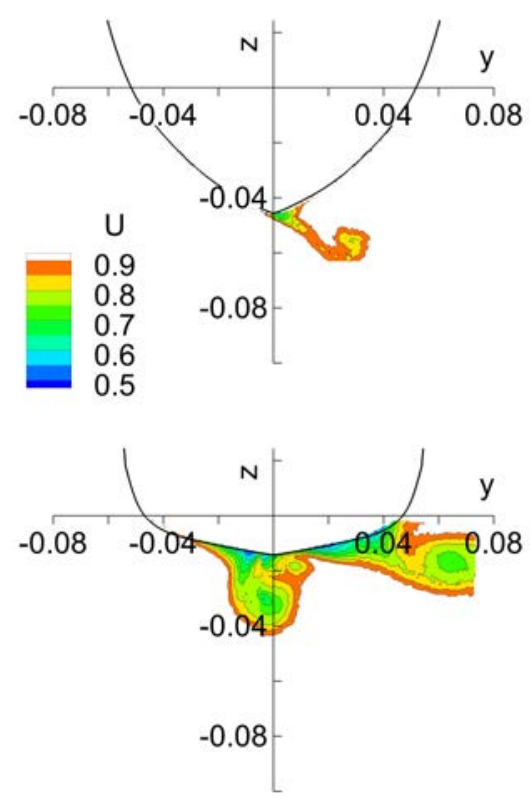

(b)
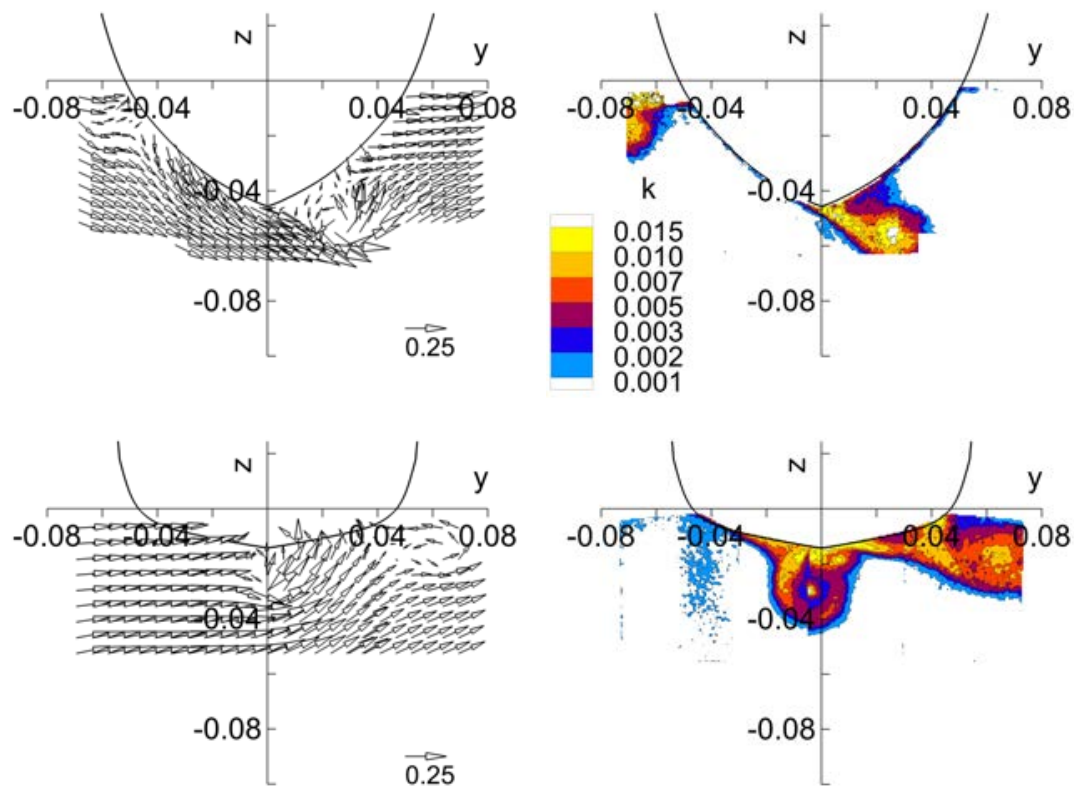

Fig. 3.(a) Pure sway at $\gamma=0^{\circ}$ : (a) axial velocity $U$ contours, (b) cross-plane $V W$-vectors, and (c) turbulent kinetic energy $k$ contours at $x=0.235$ (top) and 0.935 (bottom). The average axial velocity value computed over the region where $U \leq 0.9$ is defined as $U_{\text {mean }}$ and the average turbulent kinetic energy values computed over the regions where $k \geq 0.001$ and $k \geq 0.01$ are defined as $k_{\text {mean }}$ and $k_{\text {max }}$, respectively. 
(a)

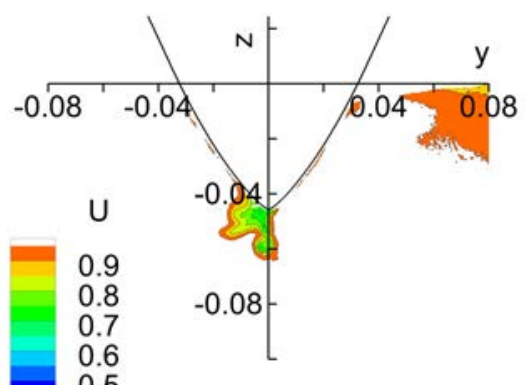

0.5
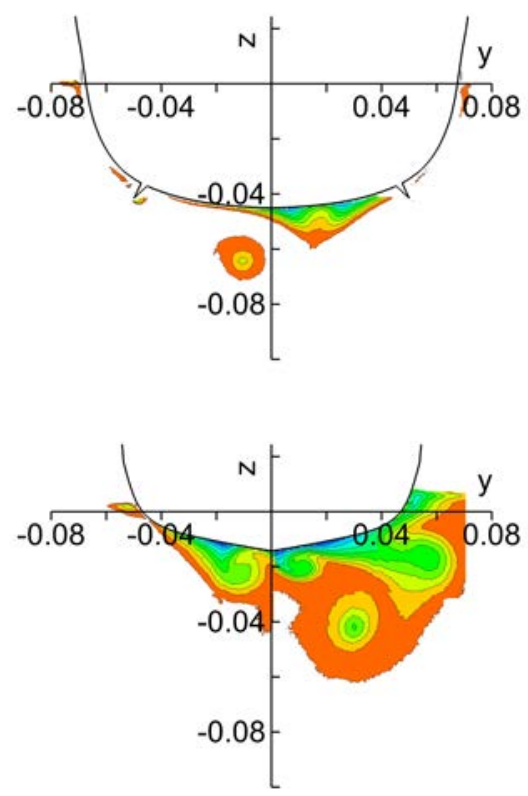

(b)
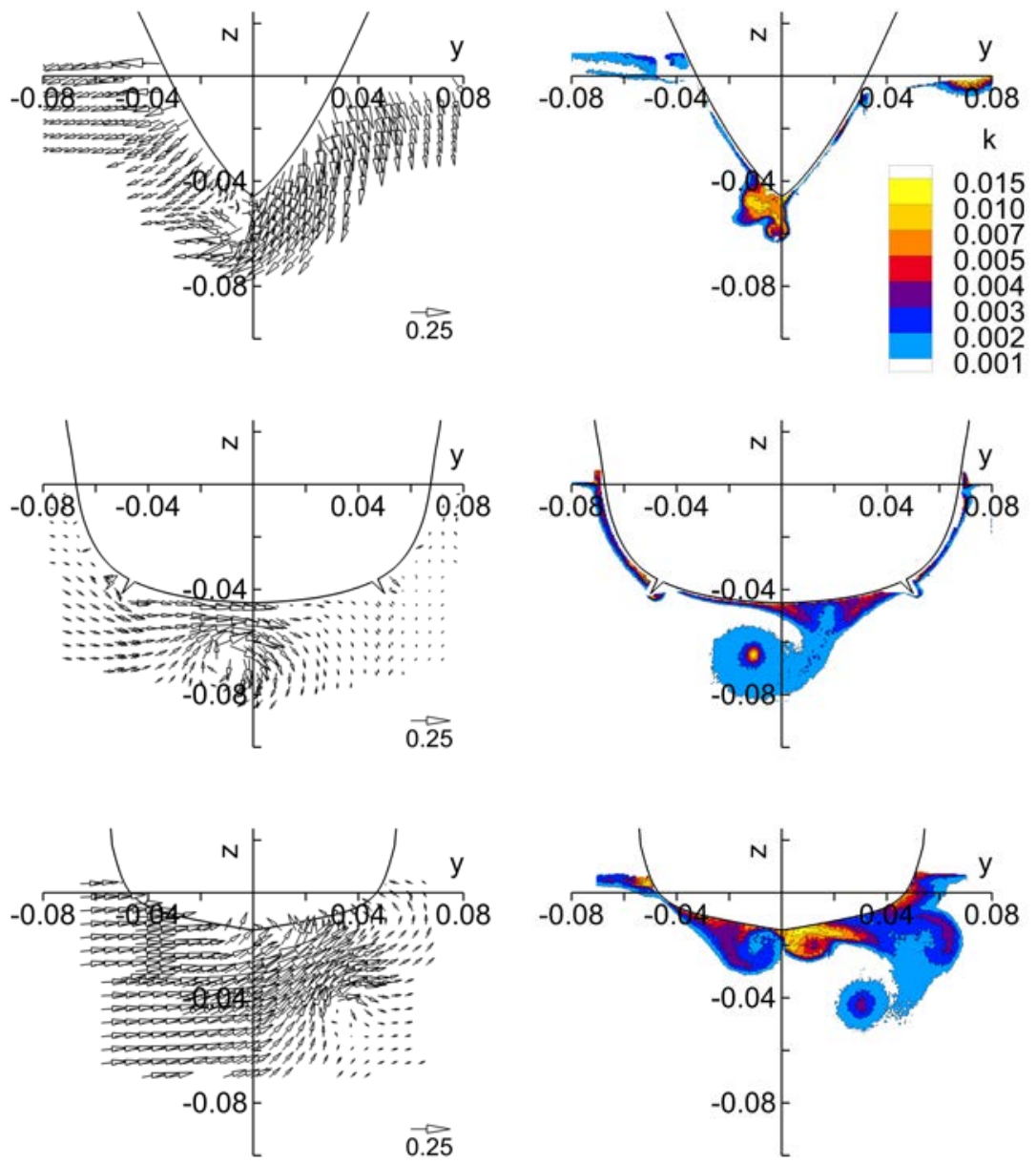

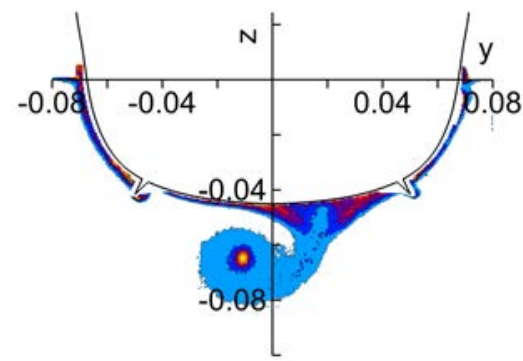

(c)

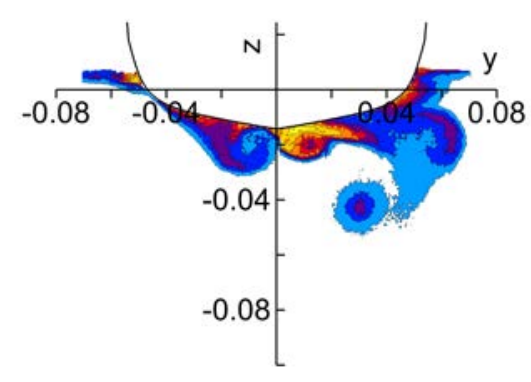

Fig. 4. Pure yaw at $\gamma=135^{\circ}$ : (a) Axial velocity $U$, (b) cross-plane $V W$-vector, and (c) turbulent kinetic energy $k$ at $x=0.135$ (top), 0.535 (middle) and 0.935 (bottom). The average axial velocity value computed over the region where $U \leq 0.9$ is defined as $U_{\text {mean }}$ and the average turbulent kinetic energy values computed over the regions where $k \geq 0.001$ and $k \geq 0.01$ are defined as $k_{\text {mean }}$ and $k_{\text {max }}$, respectively. 
(a)

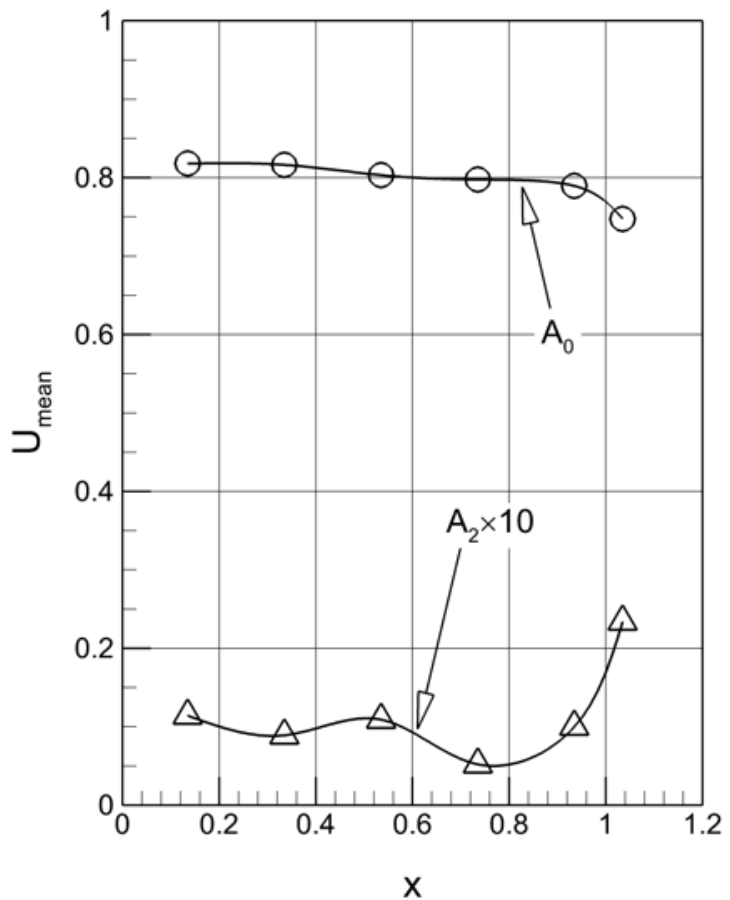

(b)

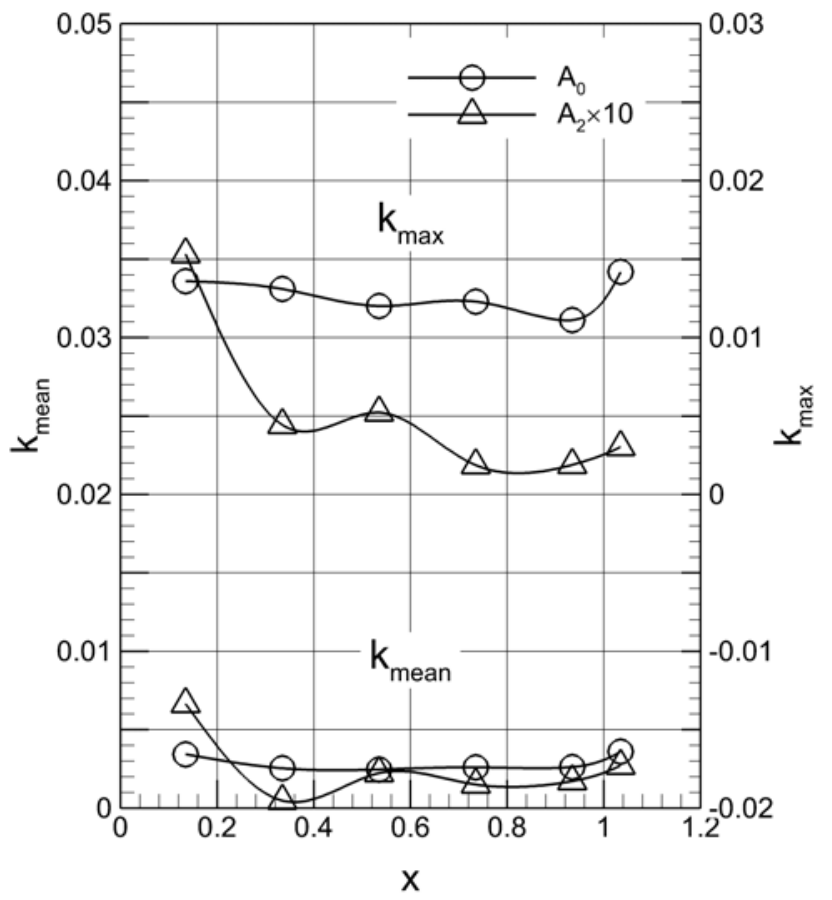

Fig. 5.Primary FS harmonics of (a) axial velocity and (b) turbulent kinetic energy for pure yaw. 
(a)

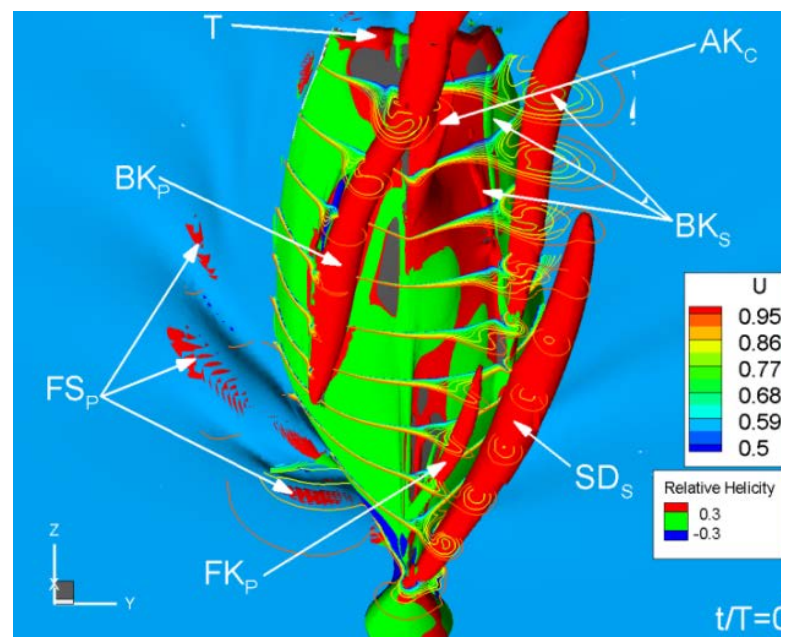

(b)

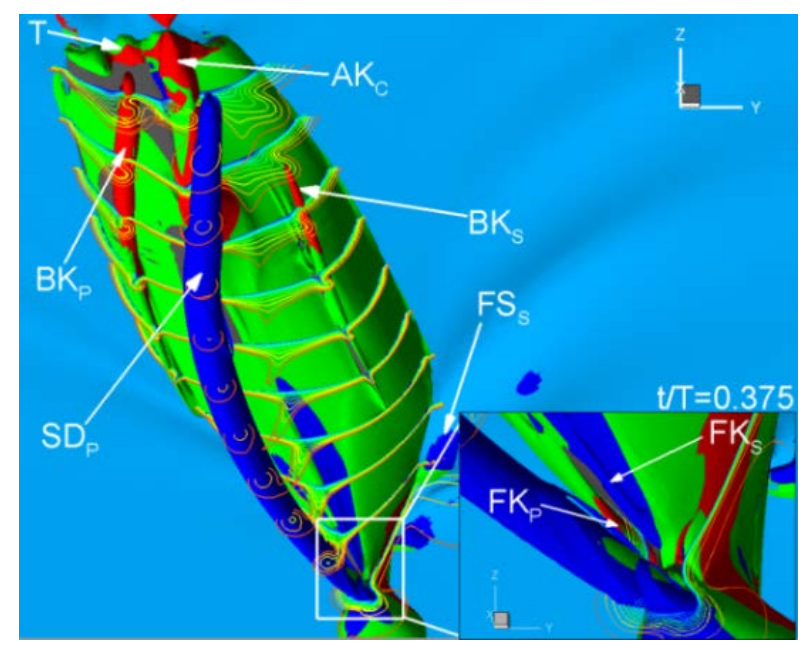

Fig. 6.Iso-Q surfaces colored by normalized helicity $H_{\mathrm{n}}$ for (a) pure sway at $t=0\left(\gamma=0^{\circ}\right)$ and (b) pure yaw at $t=0.375\left(\gamma=135^{\circ}\right)$ by CFD (Sakamoto et al., 2012). Looking upstream, a positive $H_{n}(>0.3$, red colored) signifies counter-clockwise rotation and a negative $H_{n}(<-0.3$, blue colored) signifies clockwise rotation. Additionally, the iso-contour lines of axial velocity $U$ visualize the flow boundary layers. 
(a)

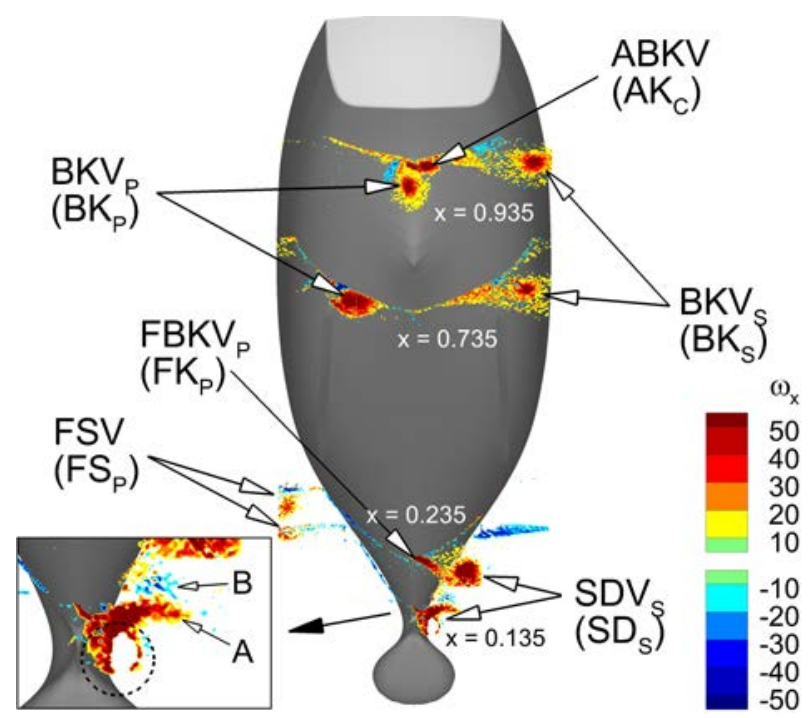

(b)

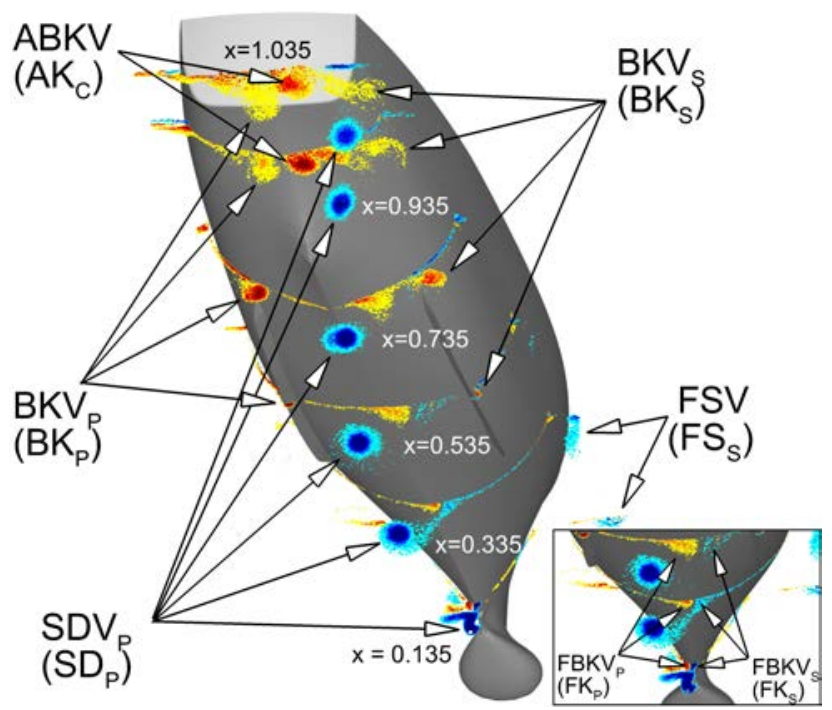

Fig. 7.Contours of $\omega_{x}$ in (a) pure sway at $t=0\left(\gamma=0^{\circ}\right)$ and (b) pure yaw at $t=0.375\left(\gamma=135^{\circ}\right)$. 
(a)

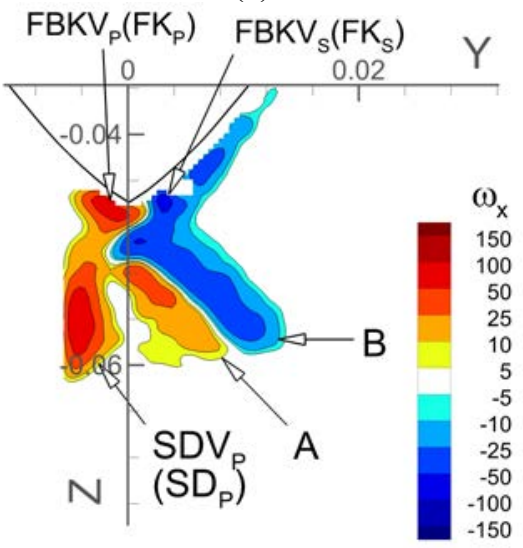

(d)

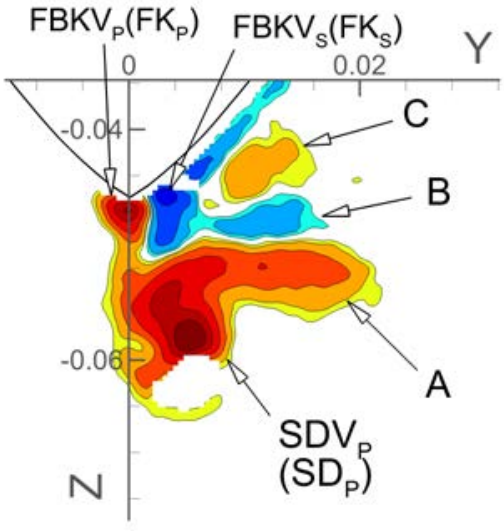

(b)

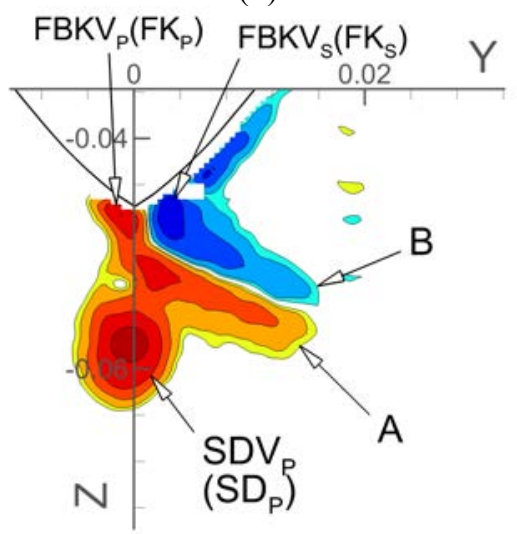

(e)

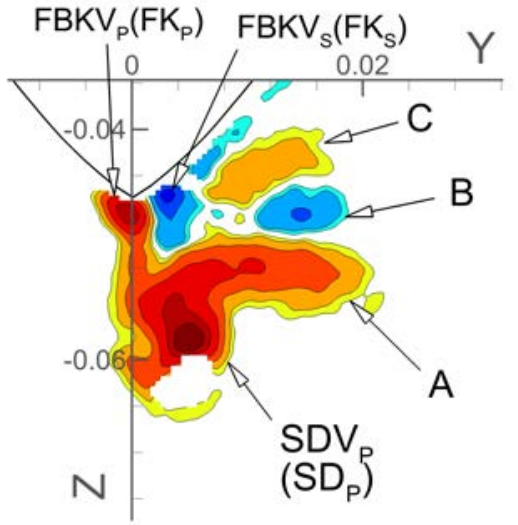

(c)

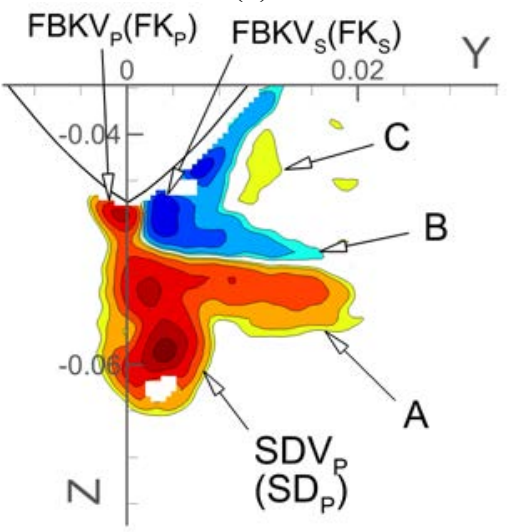

(f)

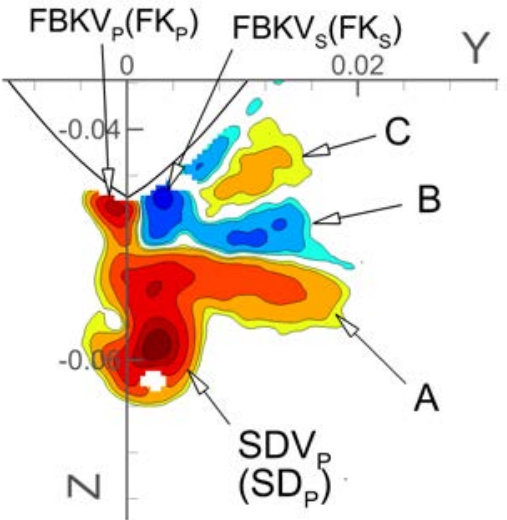

Fig. 8.Contours of $\omega_{x}$ at $x=0.135$ in pure yaw at $\gamma=$ (a) $202.5^{\circ}$, (b) $225^{\circ}$, (c) $247.5^{\circ}$, (d) $270^{\circ}$, (e) $292.5^{\circ}$, and (f) $315^{\circ}$. 
(a)

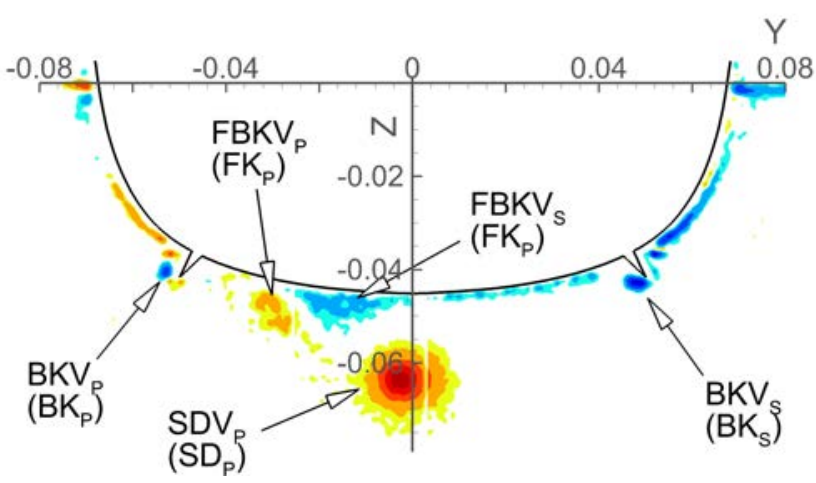

(c)

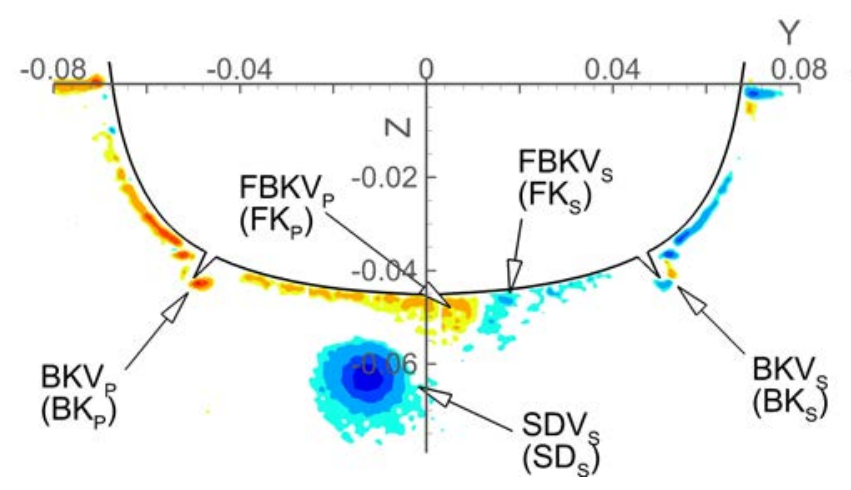

(b)

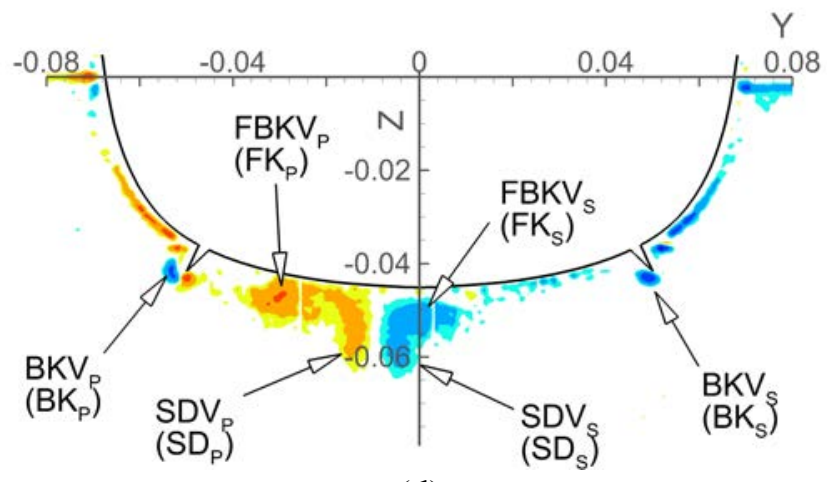

(d)

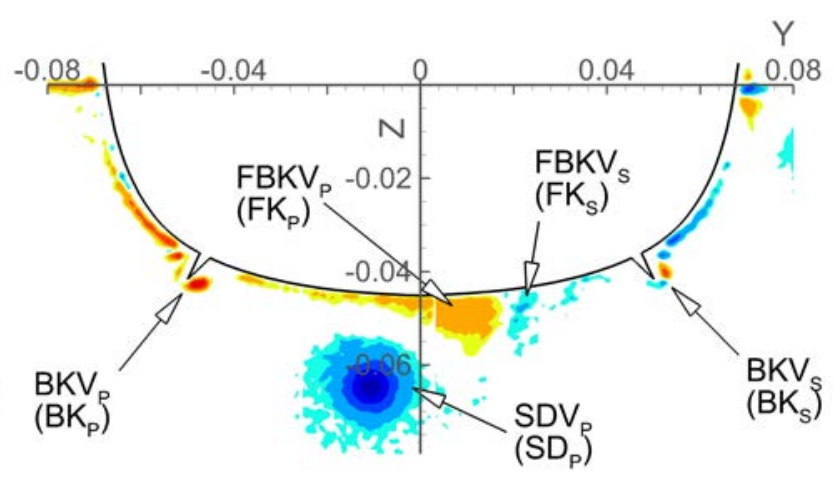

Fig. 9. Contours of $\omega_{x}$ at $x=0.535$ in pure yaw at $\gamma=$ (a) $0^{\circ}$, (b) $45^{\circ}$, (c) $90^{\circ}$ and (d) $135^{\circ}$ (See Fig. 8 for color legend). 
(a)

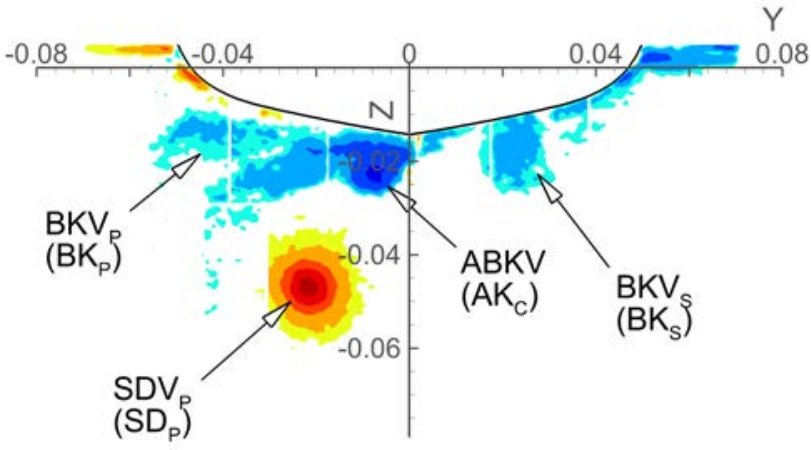

(c)

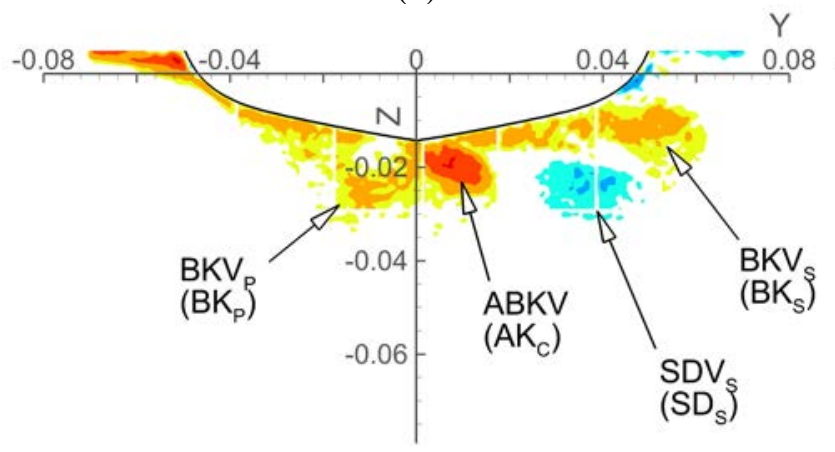

(b)

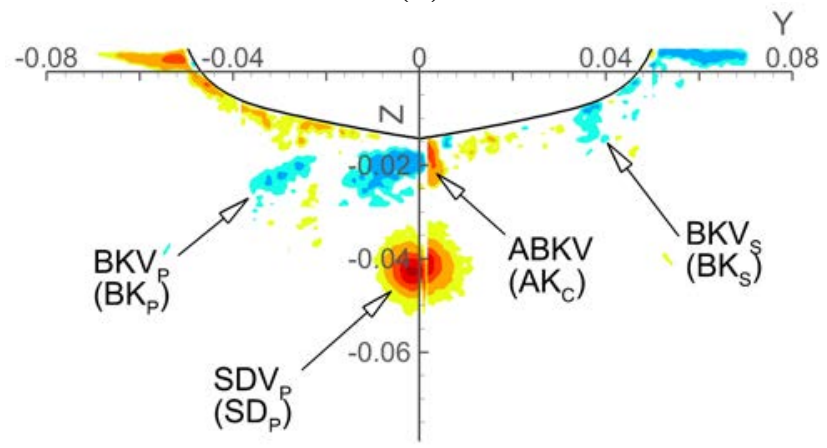

(d)

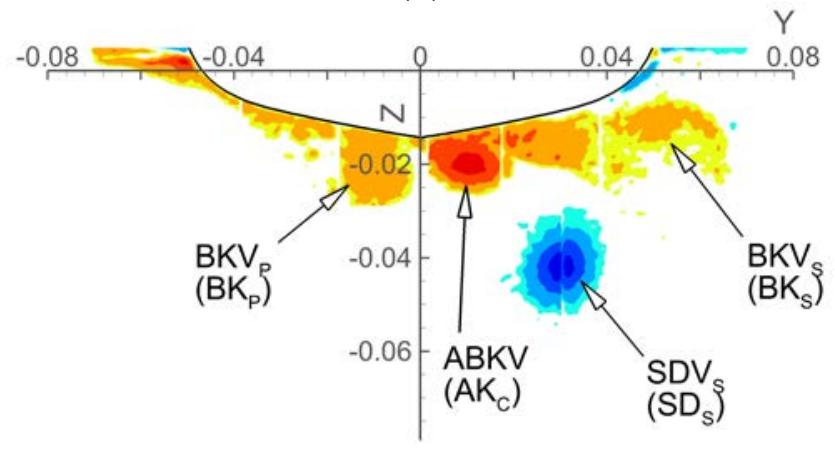

Fig. 10.Contours of $\omega_{x}$ at $x=0.935$ in pure yaw at $\gamma=$ (a) $0^{\circ}$, (b) $45^{\circ}$, (c) $90^{\circ}$ and (d) $135^{\circ}$ (See Fig. 8 for color legend). 
(a)

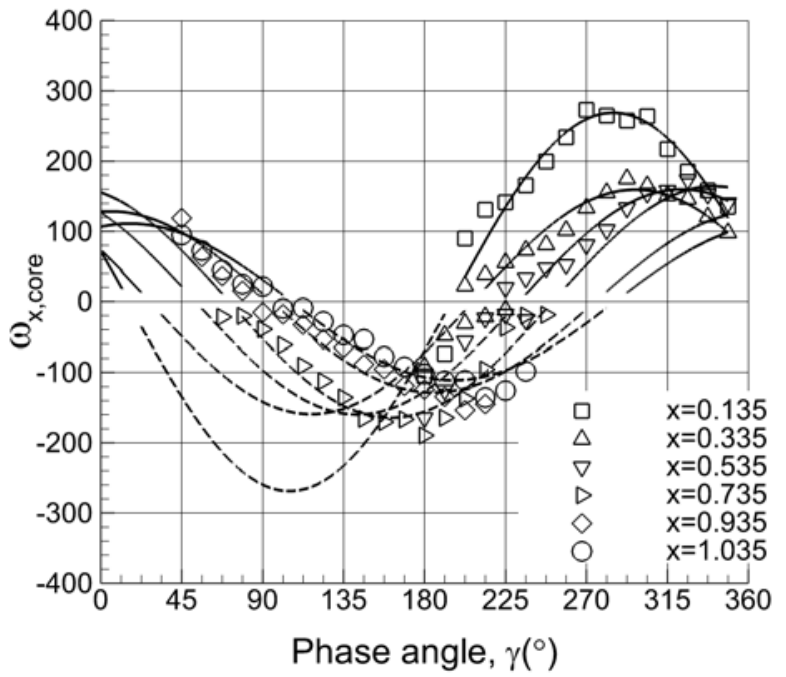

(c)

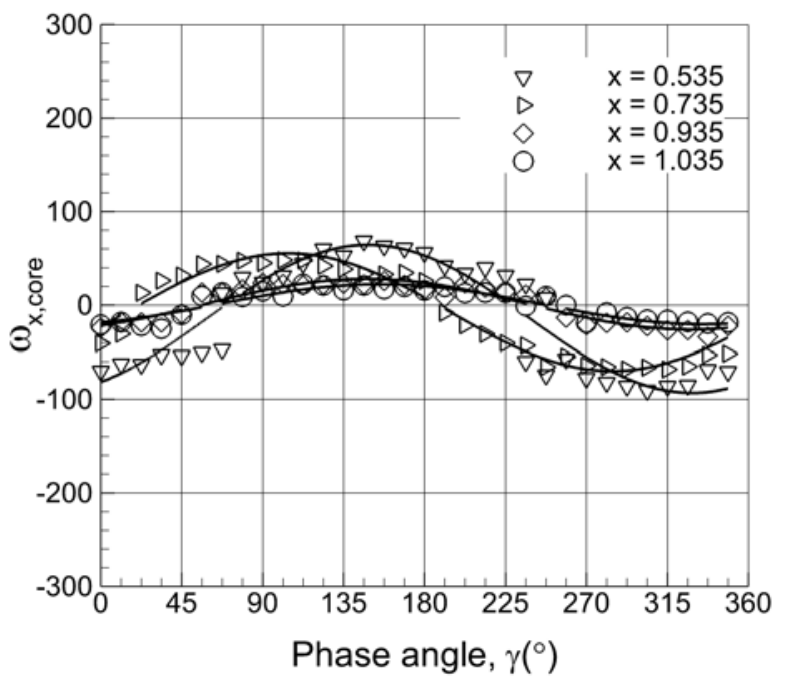

(b)

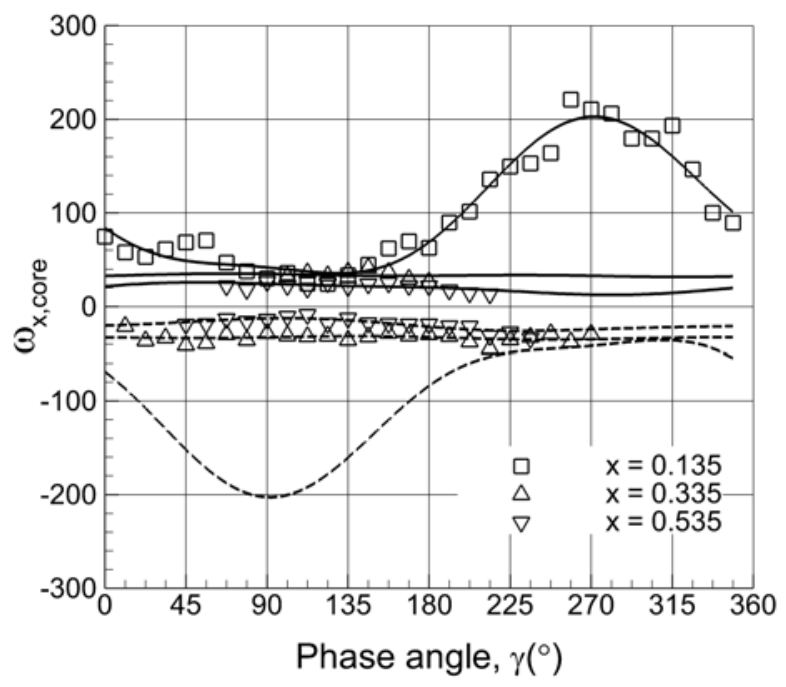

(d)

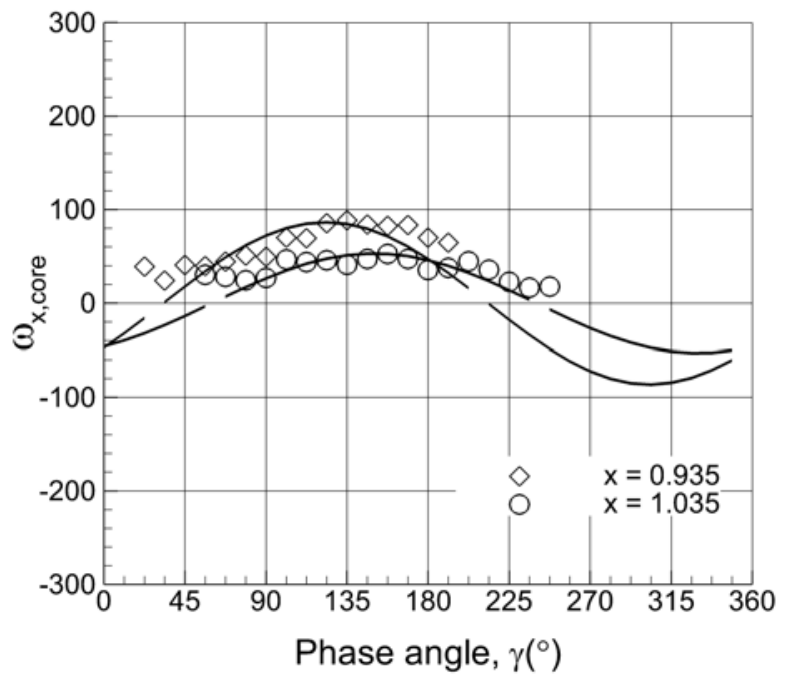

Fig. 11. Time-histories of core axial vorticity of (a) $\mathrm{SDV}_{\mathrm{P}}$ (solid lines) and $\mathrm{SDV}_{\mathrm{S}}$ (dashed lines), (b) $\mathrm{FBKV}_{\mathrm{P}}$ (solid lines) and $\mathrm{FBKV}_{\mathrm{S}}$ (dashed lines), (c) BKV $\mathrm{S}_{\mathrm{S}}$ and (d) ABKV in pure yaw. 
(a)

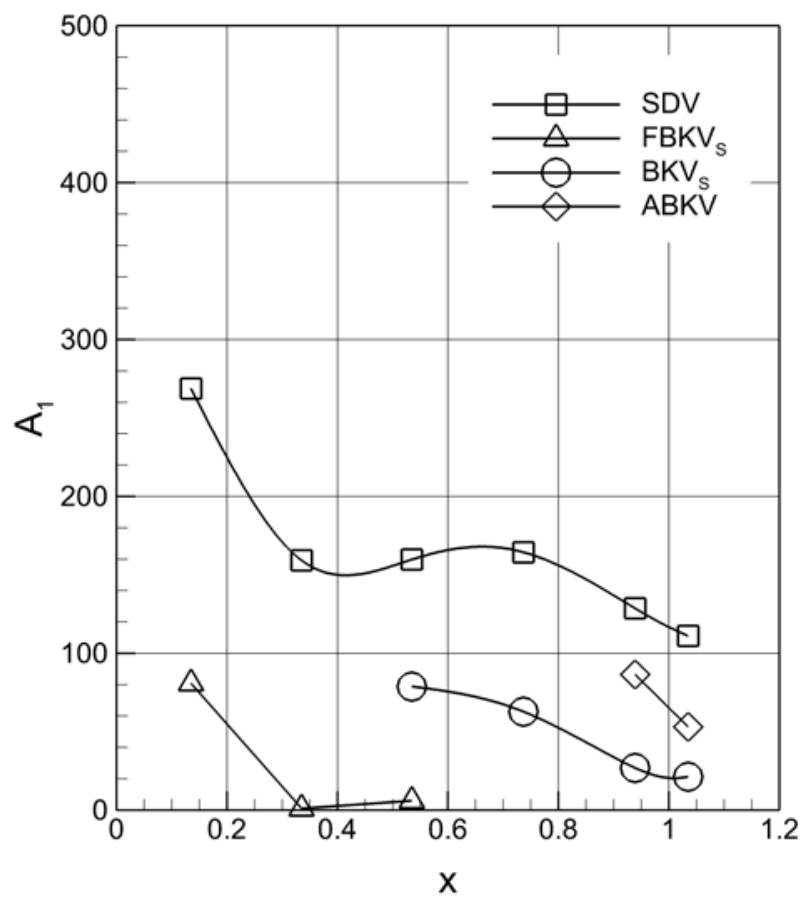

(b)

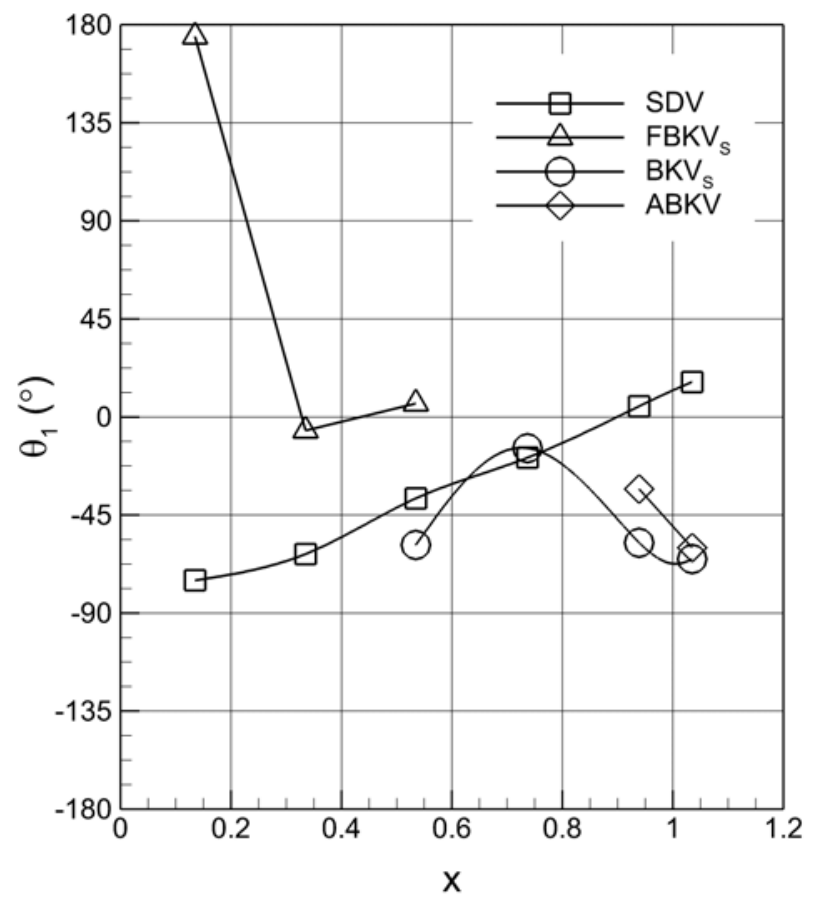

Fig. 12. The $1^{\text {st }}$-order FS harmonic (a) amplitude and (b) phase angle of $\omega_{x, \text { core }}$ in pure yaw. 
(a)

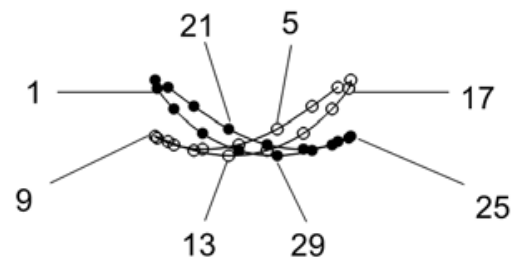

(b)

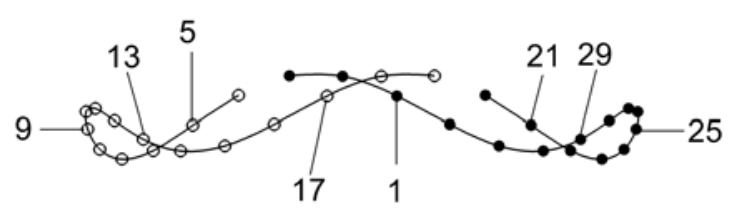

(c)

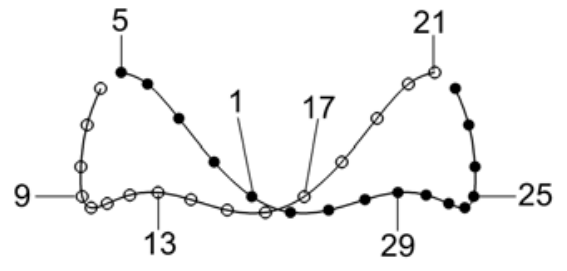

(d)
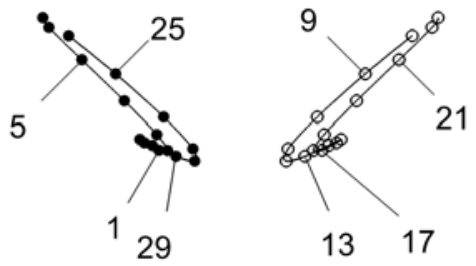

(e)

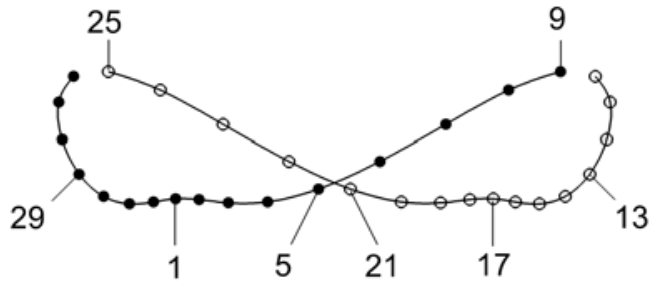

(f)

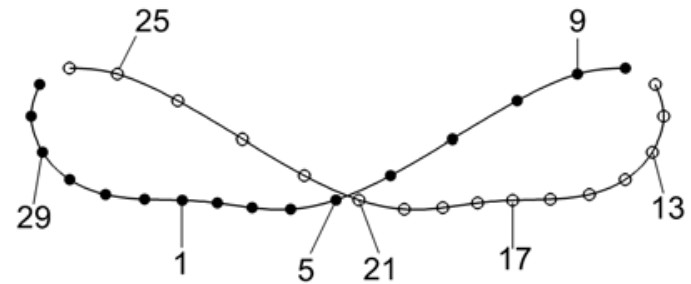

Fig. 13. Trajectories of SDV core in pure yaw: (a) $x=0.135$, (b) $x=0.335$, (c) $x=0.535$, (d) $x=0.735$, (e) $x=0.935$, and (f) $x=1.035$ (filled circle $=\mathrm{SDV}_{\mathrm{P}}$; hollow circle $=\mathrm{SDV}_{\mathrm{S}}$ ). The trajectories are not shown in scale among different $x$ locations. 
(a)

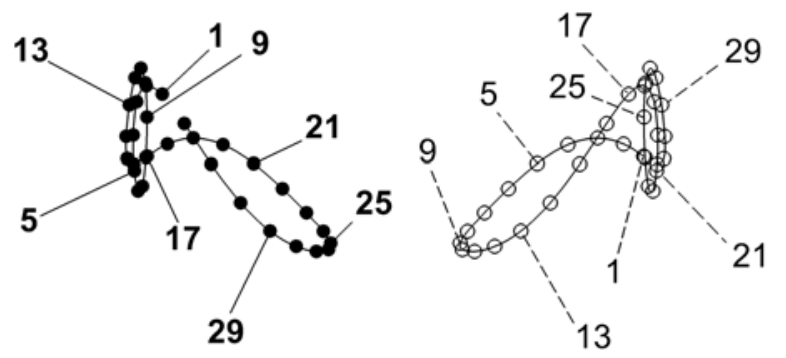

(b)

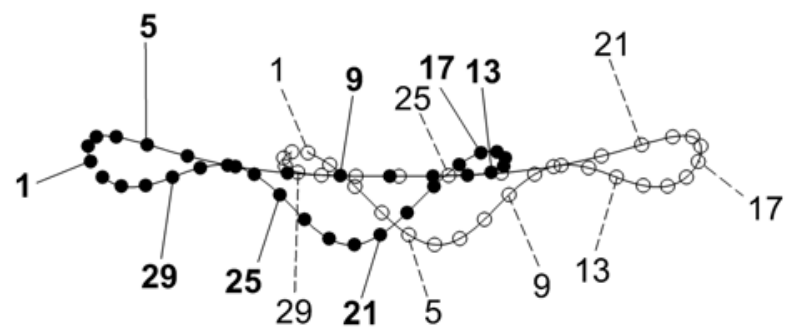

(c)

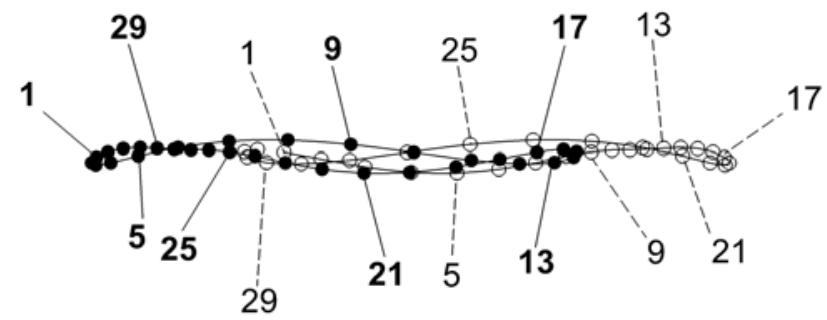

Fig. 14. Trajectories of FBKV core in pure yaw: (a) $x=0.135$, (b) $x=0.335$, and (c) $x=0.535$ (filled circle $=\mathrm{FBKV}_{\mathrm{P}}$ and hollow circle $\left.=\mathrm{FBKV}_{\mathrm{S}}\right)$. The trajectories are not shown in scale among different $x$ locations. 


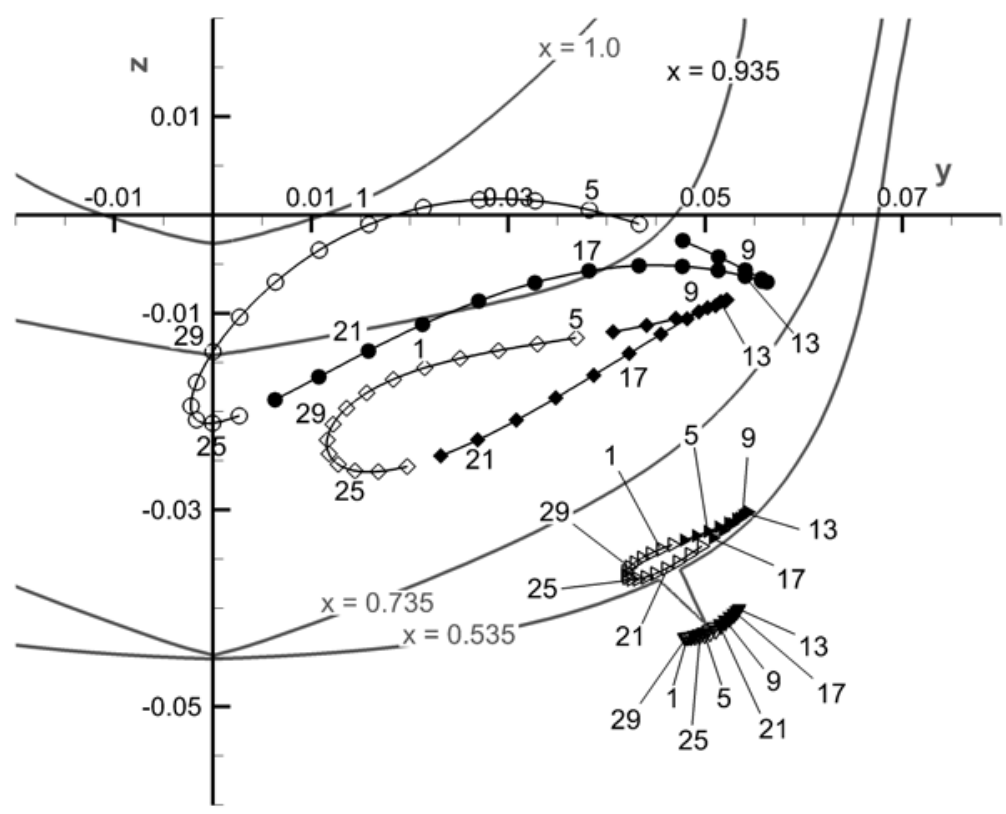

Fig. 15. Trajectories of $\mathrm{BKV}_{\mathrm{S}}$ core in pure yaw: (from bottom to top) $x=0.535,0.735,0.935$, and 1.035 (filled symbol = positive vorticity; hollow symbol = negative vorticity). 


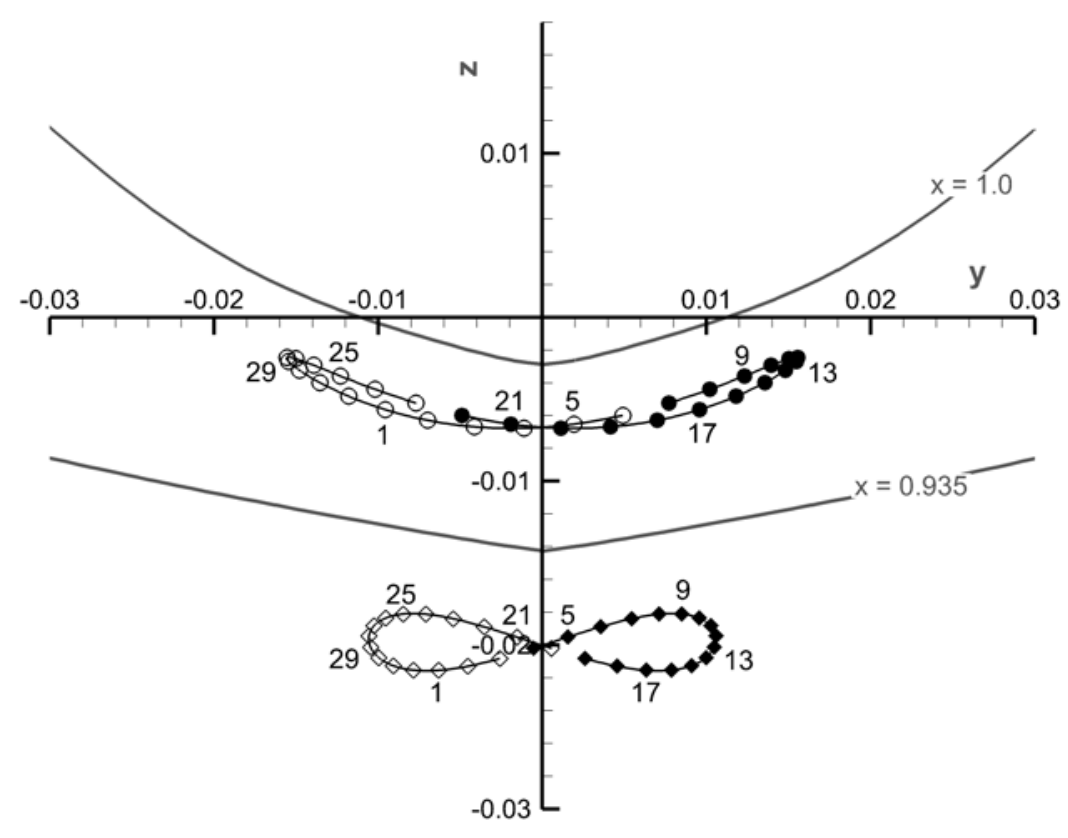

Fig. 16. Trajectories of ABKV core in pure yaw: (top) $x=0.935$ and (bottom) $x=1.035$ (filled symbol $=$ positive vorticity; hollow symbol = negative vorticity). 
(a)

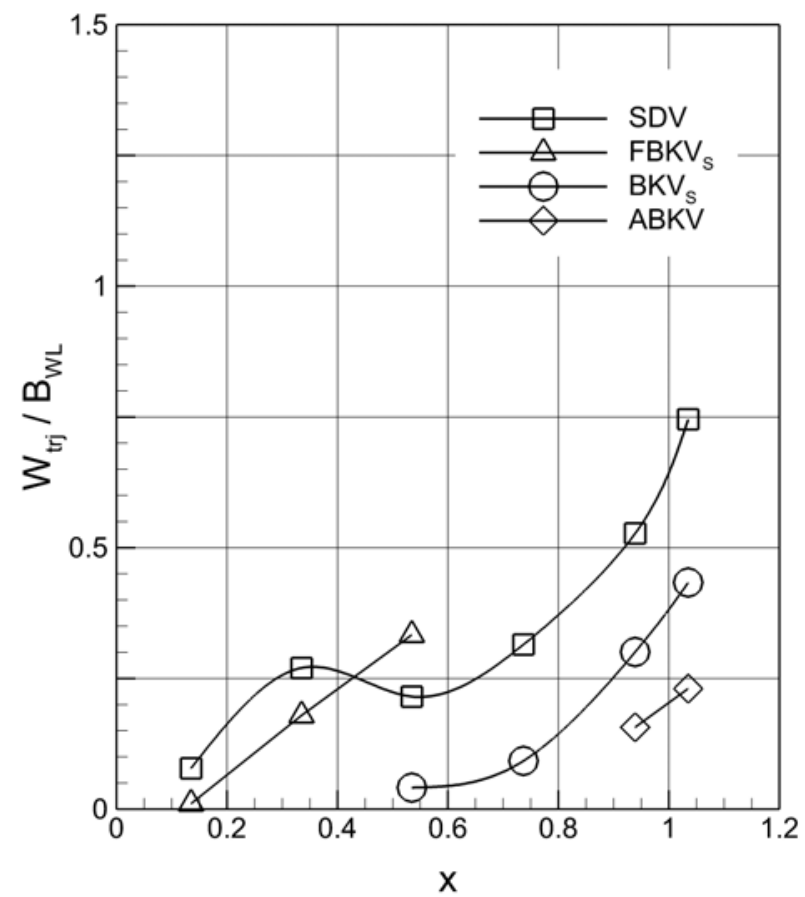

(b)

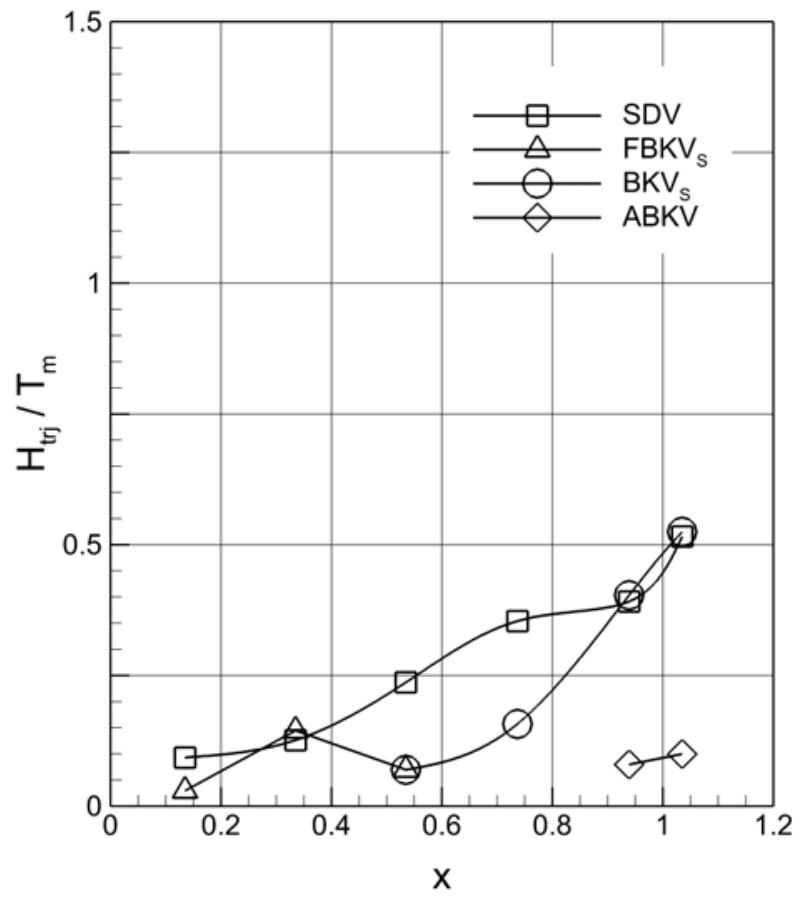

Fig. 17.Vortex core trajectories (a) width and (b) height in pure yaw. 
(a)

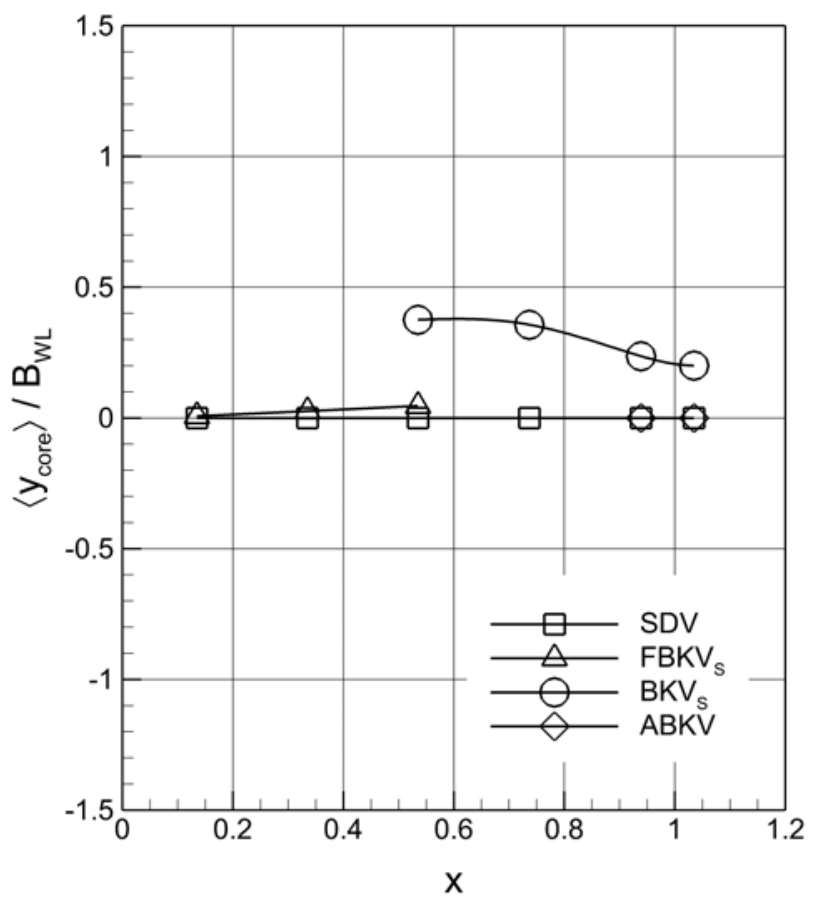

(b)

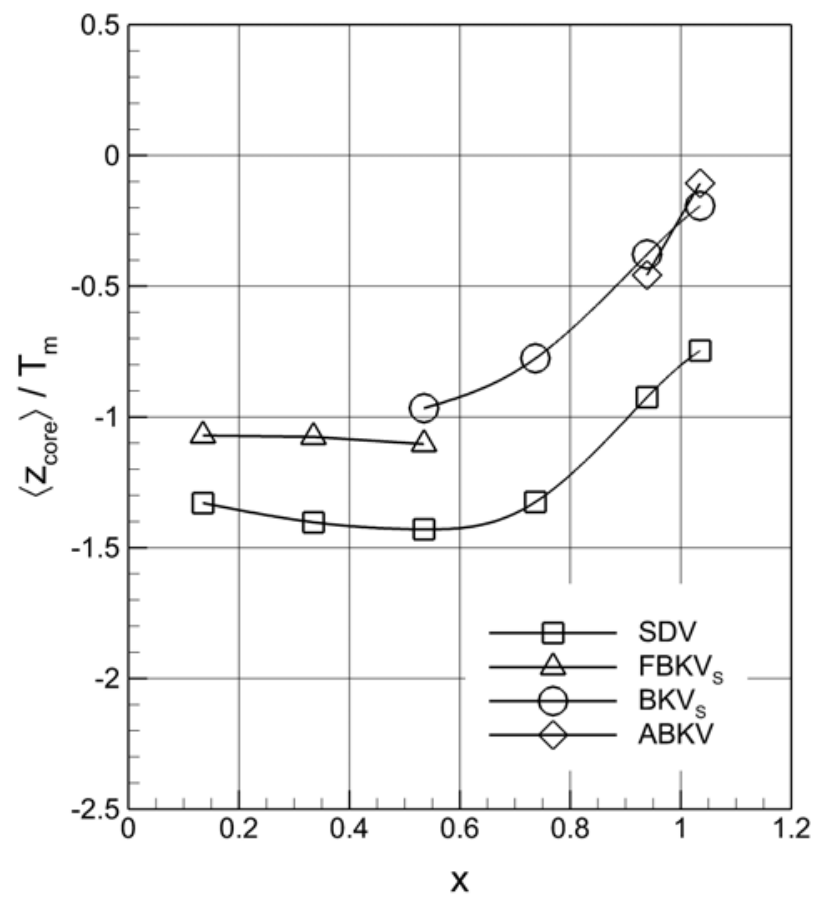

Fig. 18.Vortex core (a) mean-lateral and (b) mean-vertical displacements in pure yaw. 
(a)

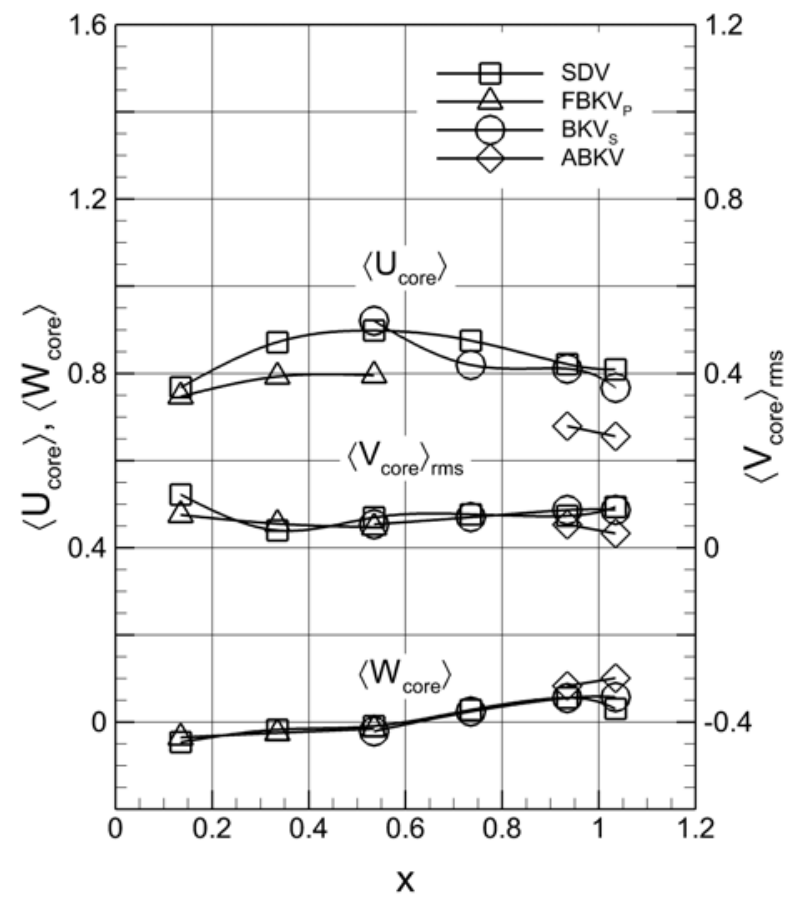

(b)

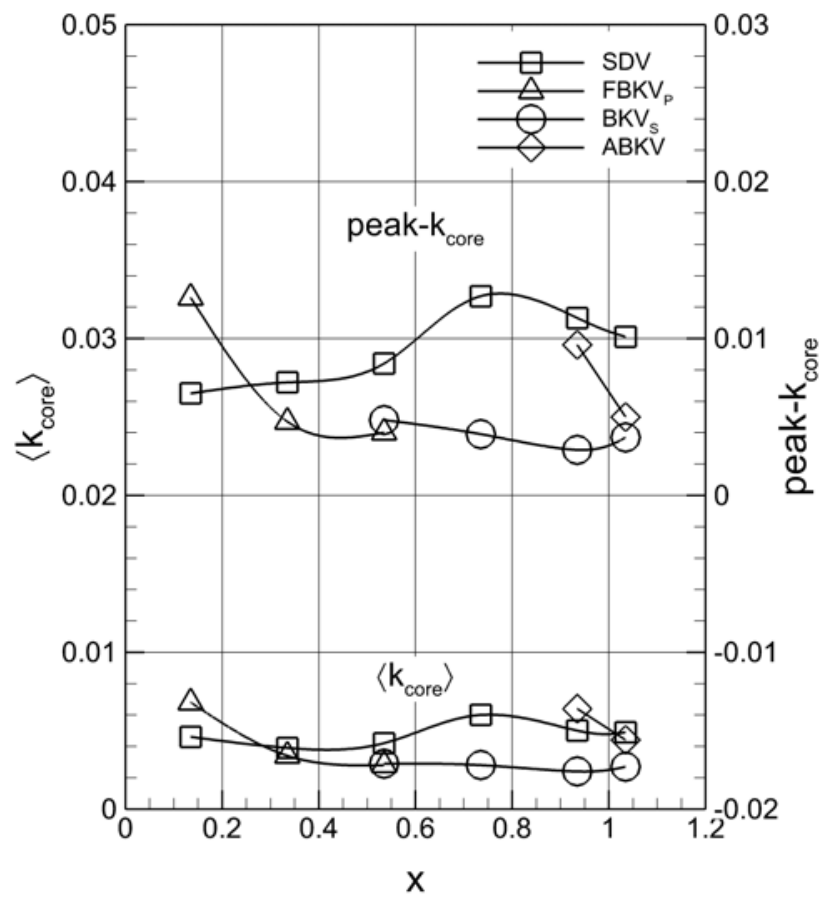

Fig. 19.Vortex core (a) velocity and (b) turbulence variables in pure yaw. 